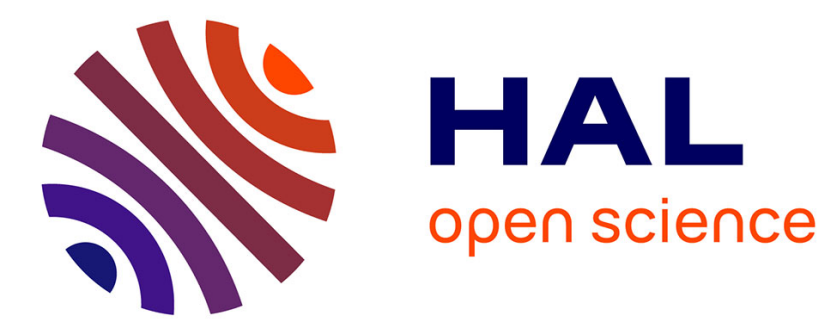

\title{
NON DISPERSIVE SOLUTIONS OF THE GENERALIZED KDV EQUATIONS ARE TYPICALLY MULTI-SOLITONS
}

Xavier Friederich

\section{- To cite this version:}

Xavier Friederich. NON DISPERSIVE SOLUTIONS OF THE GENERALIZED KDV EQUATIONS ARE TYPICALLY MULTI-SOLITONS. Annales de l'Institut Henri Poincaré C, Analyse non linéaire, In press, 10.1016/j.anihpc.2020.11.010 . hal-02888404

\section{HAL Id: hal-02888404 https://hal.science/hal-02888404}

Submitted on 3 Jul 2020

HAL is a multi-disciplinary open access archive for the deposit and dissemination of scientific research documents, whether they are published or not. The documents may come from teaching and research institutions in France or abroad, or from public or private research centers.
L'archive ouverte pluridisciplinaire HAL, est destinée au dépôt et à la diffusion de documents scientifiques de niveau recherche, publiés ou non, émanant des établissements d'enseignement et de recherche français ou étrangers, des laboratoires publics ou privés. 


\title{
NON DISPERSIVE SOLUTIONS OF THE GENERALIZED KDV EQUATIONS ARE TYPICALLY MULTI-SOLITONS
}

\author{
XAVIER FRIEDERICH
}

\begin{abstract}
Aвstract. We consider solutions of the generalized Korteweg-de Vries equations (gKdV) which are non dispersive in some sense (in the spirit of [18]) and which remain close to multisolitons. We show that these solutions are necessarily pure multi-solitons. For the Korteweg-de Vries equation $(\mathrm{KdV})$ and the modified Korteweg-de Vries equation $(\mathrm{mKdV})$ in particular, we obtain a characterization of multi-solitons and multi-breathers in terms of non-dispersion.
\end{abstract}

\section{INTRODUCTION}

1.1. Setting of the problem and known results. We consider the generalized Kortewegde Vries equations

$(\mathrm{gKdV})$

$$
\left\{\begin{array}{l}
\partial_{t} u+\partial_{x}\left(\partial_{x}^{2} u+u^{p}\right)=0 \\
u(0)=u_{0} \in H^{1}(\mathbb{R})
\end{array}\right.
$$

where $(t, x)$ are elements of $\mathbb{R} \times \mathbb{R}$ and $p>1$ is an integer.

Recall that the Cauchy problem for ( $\mathrm{gKdV})$ is locally well-posed in $H^{1}(\mathbb{R})$ from a standard result by Kenig, Ponce and Vega [9] and that the two following quantities are conserved for each solution $u$ of $(\mathrm{gKdV})$ for all $t$ :

- (the $L^{2}$-mass) $\int_{\mathbb{R}} u^{2}(t, x) d x$

- (the energy) $\int_{\mathbb{R}}\left\{\frac{1}{2} u_{x}^{2}-\frac{1}{p+1} u^{p+1}\right\}(t, x) d x$.

In addition, the set of solutions of $(\mathrm{gKdV})$ is conserved under scaling transformation

$$
u \longmapsto\left((t, x) \mapsto \lambda^{\frac{2}{p-1}} u\left(\lambda^{3} t, \lambda x\right)\right),
$$

for all $\lambda>0$, and the $\dot{H}^{\sigma(p)}$-norm is invariant under this transformation, where $\sigma(p):=$ $\frac{1}{2}-\frac{2}{p-1}$. Let us recall that the global dynamics of the solutions depends on the sign of $\sigma(p)$. The case $\sigma(p)<0$ that is $1<p<5$, is called $L^{2}$-subcritical, and all $H^{1}$-solutions of $(\mathrm{gKdV})$ are then global (in time) and $H^{1}$-uniformly bounded. If $\sigma(p)=0$, that is $p=5$, we are in $L^{2}$-critical case and solutions might blow up in finite time $[16,17,23,24,25]$. In the $L^{2}$ supercritical case, corresponding to $\sigma(p)>0$ (or $p>5$ ), much less is known but finite time blow up is expected: existence of $p^{*}>5$ and of blow-up solutions for all $p \in\left(5, p^{*}\right)$ are proven in [10].

Moreover it is well-known that ( $\mathrm{gKdV})$ admits a family of explicit traveling wave solutions indexed by $\mathbb{R}_{+}^{*} \times \mathbb{R}$. Let $Q$ be the unique (up to translation) positive solution in $H^{1}(\mathbb{R})$ (known also as ground state) to the following stationary elliptic problem associated with ( $\mathrm{gKdV})$

$$
Q^{\prime \prime}+Q^{p}=Q
$$

2010 Mathematics Subject Classification: Primary 35B53, 35Q53; Secondary 35B40, 35B65.

Key words: generalized KdV equations; solitons; multi-solitons. 
given by the explicit formula

$$
Q(x)=\left(\frac{p+1}{2 \operatorname{ch}^{2}\left(\frac{p-1}{2} x\right)}\right)^{\frac{1}{p-1}} .
$$

Then for all $c_{0}>0$ (velocity parameter) and $x_{0} \in \mathbb{R}$ (translation parameter),

$$
R_{c_{0}, x_{0}}(t, x)=Q_{c_{0}}\left(x-c_{0} t-x_{0}\right)
$$

is a global traveling wave solution of $(\mathrm{gKdV})$ classically named soliton solution, where $Q_{c_{0}}(x)=$ $c_{0}^{\frac{1}{p-1}} Q\left(\sqrt{c_{0}} x\right)$. It is orbitally stable if and only if $p<5$ ( $L^{2}$-subcritical case) (see Weinstein [32], Bona, Souganidis and Strauss [2], Grillakis, Shatah and Strauss [8], and Martel and Merle [15]).

Solitons are special objects which enjoy very specific properties. Let us recall the following rigidity result, which roughly asserts that non dispersive solutions to ( $\mathrm{gKdV}$ ) which are close to solitons are actually exactly solitons.

Theorem 1.1 (Liouville property near a soliton; Martel and Merle [13, 19]). Let $c_{0}>0$. There exists $\alpha>0$ such that if $u \in \mathscr{C}\left(\mathbb{R}, H^{1}(\mathbb{R})\right)$ is a solution of $(g K d V)$ satisfying, for some $\mathscr{C}^{1}$ function $y: \mathbb{R} \rightarrow \mathbb{R}$,

$$
\text { (closeness to a soliton) } \quad \forall t \in \mathbb{R}, \quad\left\|u(t, \cdot+y(t))-Q_{c_{0}}\right\|_{H^{1}} \leq \alpha,
$$

(1.3) (non-dispersion) $\forall \varepsilon>0, \exists R>0, \forall t \in \mathbb{R}, \quad \int_{|x|>R} u^{2}(t, x+y(t)) d x \leq \varepsilon$,

then there exist $c_{1}>0, x_{1} \in \mathbb{R}$ such that

$$
\forall t, x \in \mathbb{R}, \quad u(t, x)=Q_{c_{1}}\left(x-x_{1}-c_{1} t\right) .
$$

This striking result has its own interest of course, but we emphasize that it is also a key ingredient to prove asymptotic stability of $(\mathrm{gKdV})$ solitons (we refer to $[13,14,19])$. We highlight the fact that this result applies in each mass subcritical, critical, and supercritical case by requiring the solution $u$ to remain close to a soliton (up to translation) for all times (1.2). In the $L^{2}$-subcritical case where solitons are known to be stable, (1.2) can be relaxed to hold only at $t=0$.

Finally let us note that solitons play a fundamental role in the study and the understanding of the $(\mathrm{gKdV})$ flow; the important soliton resolution conjecture asserts that any solution with generic initial condition behaves as a sum of solitons plus a radiative-dispersive term as time goes to infinity. In this spirit, built upon solitons, we are interested in other solutions to our problem, namely multi-soliton solutions, defined as follows.

Definition 1.2. Let $N \geq 1$ and consider $N$ solitons $R_{c_{i}, x_{i}}$ as in (1.1) with speeds $0<c_{1}<$ $\cdots<c_{N}$. A multi-soliton in $+\infty$ (resp. in $\left.-\infty\right)$ associated with the $R_{c_{i}, x_{i}}$ is an $H^{1}$-solution $u$ of $(g K d V)$ defined in a neighborhood of $+\infty$ (resp. $-\infty)$ and such that

$$
\left\|u(t)-\sum_{i=1}^{N} R_{c_{i}, x_{i}}(t)\right\|_{H^{1}} \rightarrow 0, \quad \text { as } t \rightarrow+\infty(\text { resp. as } t \rightarrow-\infty) .
$$

Multi-solitons are known to exist for all $p>1$; they are even explicit for $p=2(\mathrm{KdV})$ [27, section 16] and for $p=3(\mathrm{mKdV})$ [30, Chapter 5, formula (5.5)]. What is more, the classification of the multi-solitons of $(\mathrm{gKdV})$ is complete. Let us gather the main results. 
Theorem 1.3 (Martel [12]; Côte, Martel and Merle [6]; Combet [5]). Let $p>1$ be an integer and let $N \geq 1,0<c_{1}^{+}<\cdots<c_{N}^{+}$, and $x_{1}^{+}, \ldots, x_{N}^{+} \in \mathbb{R}$.

If $p \leq 5$, there exists $T_{0} \geq 0$ and a unique multi-soliton $u \in \mathscr{C}\left(\left[T_{0},+\infty\right), H^{1}(\mathbb{R})\right)$ associated with the $R_{c_{i}^{+}, x_{i}^{+}}, i \in\{1, \ldots, N\}$.

If $p>5$, there exists a one-to-one map $\Phi$ from $\mathbb{R}^{N}$ to the set of all $H^{1}$-solutions of $(g K d V)$ defined in a neighborhood of $+\infty$ such that $u$ is a multi-soliton in $+\infty$ associated with the $R_{C_{i}^{+}, x_{i}^{+}}$if and only if there exist $\lambda \in \mathbb{R}^{N}$ and $T_{0} \geq 0$ such that $u_{\mid\left[T_{0},+\infty\right)}=\Phi(\lambda)_{\mid\left[T_{0},+\infty\right)}$.

Moreover, in each case, $u$ belongs to $\mathscr{C}\left(\left[T_{0},+\infty\right), H^{s}(\mathbb{R})\right)$ for all $s \geq 0$, and there exist $\theta>0$ and positive constants $C_{s}$ such that for all $s \geq 0$, for all $t \geq T_{0}$,

$$
\left\|u(t)-\sum_{i=1}^{N} R_{c_{i}^{+}, x_{i}^{+}}(t)\right\|_{H^{s}} \leq C_{s} e^{-\theta t} .
$$

In the $L^{2}$-subcritical case (like solitons), sums of decoupled and ordered solitons are stable in $H^{1}(\mathbb{R})$, even asymptotically stable (Martel, Merle and Tsai [26] and Martel and Merle [19]), and so do multi-solitons.

1.2. Main results. Several properties available for solitons have been adapted or even extended to multi-solitons. This article precisely takes this step since it aims at providing an analogue of the rigidity property of Theorem 1.1 in the multi-soliton case. We consider solutions of $(\mathrm{gKdV})$ that are non dispersive in some sense and uniformly close to the sum of $N$ solitons, and show that they are exact multi-solitons.

Theorem 1.4 (Liouville property near a multi-soliton). Let $u$ be a solution of $(g K d V)$ which belongs to $\mathscr{C}\left([0,+\infty), H^{1}(\mathbb{R})\right)$. Assume the existence of $\rho>0$ such that

$$
\forall \varepsilon>0, \exists R_{\varepsilon}>0, \forall t \geq 0, \quad \int_{x<\rho t-R_{\varepsilon}} u^{2}(t, x) d x \leq \varepsilon .
$$

Let $N \geq 1$ be an integer and consider $N$ positive real numbers $0<c_{1}<\cdots<c_{N}$. There exists $\alpha=\alpha\left(c_{1}, \ldots, c_{N}, \rho\right)>0$ such that the following holds: if there exist $N$ functions $x_{1}, \ldots, x_{N}: \mathbb{R}^{+} \rightarrow \mathbb{R}$ of class $\mathscr{C}^{1}$ satisfying

$$
\forall t \geq 0, \quad\left\|u(t)-\sum_{i=1}^{N} Q_{c_{i}}\left(\cdot-x_{i}(t)\right)\right\|_{H^{1}} \leq \alpha,
$$

and

$$
\forall t \geq 0, \forall i \in\{1, \ldots, N-1\}, \quad x_{i+1}(t)-x_{i}(t) \geq|\ln \alpha|,
$$

then $u$ is a multi-soliton (in $+\infty$ ). In other words, there exist $\theta>0,0<c_{1}^{+}<\cdots<c_{N}^{+}$, $x_{1}^{+}, \ldots, x_{N}^{+} \in \mathbb{R}$ and positive constants $C_{s}$ such that for all $t \geq 0$, (1.5) is granted.

Remark 1.5. In the $L^{2}$-subcritical case $1<p<5$, as sum of decoupled solitons are stable, assumptions (1.7) and (1.8) can be relaxed to hold only at time $t=0$.

This result is a natural extension of Theorem 1.1 to multi-solitons in $+\infty$, which are the only solutions which are non dispersive in the sense (1.6) (and remain close to a sum of solitons): this is a nice dynamical characterization of multi-solitons among solutions to $(\mathrm{gKdV})$. By contraposition, it means that if a solution $u$ remains in large time sufficiently close to a multisoliton but is not a multi-soliton, then it disperses insofar as (1.6) fails.

We emphasize that, in contrast with the original statement for one soliton (Theorem 1.1), the non dispersion assumption (1.6) requires the mass to be located essentially for $x \geq \rho t$ for some small positive speed $\rho>0$ (and almost touches $x=0$ ); it allows (seemingly) for much more room than in the condition (1.3), which requires that the mass be essentially concentrated in a moving ball of fixed size $R_{\varepsilon}$. 
Furthermore, the assumptions in Theorem 1.4 are done only for positive times $t \geq 0$ (and not for all times $t \in \mathbb{R}$ ). As it applies of course to the case of a single soliton, Theorem 1.4 actually extends and refines Theorem 1.1.

We must underline that this improvement to focus on the behavior for positive times only is actually very meaningful. Indeed, in view of the above result, a solution which would be non dispersive at times $+\infty$ and $-\infty$ would be a multi-soliton at both ends: but such a behavior is not to be expected, except in the integrable cases of $p=2(\mathrm{KdV})$ and $p=3(\mathrm{mKdV})$ and the Gardner nonlinearity $u^{2}-\lambda u^{3}$. To support this, let us refer to the work by Martel and Merle [20, 21, 22] (see also Muñoz [28]) on the description of 2-solitons: starting with a 2-soliton solution at $-\infty$ (for the quartic $p=4(\mathrm{gKdV})$ ), the collision is almost but not elastic, and there is a non zero defect (which one can quantify), so that it is not a 2-soliton at $+\infty$ (and so, by Theorem 1.4, it must be dispersive in the sense of (1.6)).

In principle, the computations in the articles above could extend to $N$-solitons for $N \geq 3$, but it has not been performed yet, and one could still wonder if there is always a defect. If one is willing to assume non dispersion for all time $t \in \mathbb{R}$, our conclusion is that the solution under consideration is a multi-soliton in $+\infty$ for which all derivatives decay exponentially in space for each fixed values of $t$. More precisely, we have

Corollary 1.6. Let $u \in \mathscr{C}\left(\mathbb{R}, H^{1}(\mathbb{R})\right)$ satisfy the assumptions (1.7), (1.8) of Theorem 1.4, and assume (to replace (1.6)) the existence of two constants $0<\sigma<\rho$ such that

$$
\forall \varepsilon>0, \exists R_{\varepsilon}>0, \forall t \in \mathbb{R}, \quad \int_{\mathscr{B}\left(\rho t, \sigma|t|+R_{\varepsilon}\right)^{C}} u^{2}(t, x) d x \leq \varepsilon .
$$

Then the conclusion of Theorem 1.4 holds, and also the following exponential decay property at fixed time, for all $s \in \mathbb{N}$, and for some possibly larger constant $C_{s}$ :

$$
\forall t \geq 0, \forall x \in \mathbb{R}, \quad\left|\partial_{x}^{s} u(t, x)\right| \leq C_{s} \sum_{i=1}^{N} e^{-\theta\left|x-c_{i}^{+} t\right|} .
$$

As it was first observed in [13], non dispersion (for all times) actually self improves to smoothness and exponential decay in space (outside the center of mass). Of course, this is very relevant for solitons, which exhibit precisely this spatial behavior. But it has yet to be proven that multi-solitons do have spatial exponential decay (1.10) as well; even though it is a very natural conjecture, and that it is known that multi-solitons are smooth. To be able to conclude to (1.10), one has currently to make the assumption (1.9) (in fact, it would be sufficient to assume that $u$ and $u(-t)$ satisfy (1.6) and to assume in addition that the analog of (1.6) with $x>(\rho+\sigma) t+R_{\mathcal{E}}$ holds for positive times), and for the time being, the above Corollary 1.6 is meaningful.

Remark 1.7. In the $L^{2}$-subcritical case $1<p<5$, assumptions (1.7) and (1.8) can be relaxed to hold only a time $t=0$. If they hold for large enough times, positive and negative (or outside of the collision period), the conclusion can be strengthened to $u$ being a multi-soliton at $+\infty$ and $-\infty$, and satisfying (1.10) for all $t \in \mathbb{R}$.

In the context of the particular $(\mathrm{KdV})$ equation (corresponding to $p=2$ ), we claim next a result which gives rise to a simplified characterization of multi-solitons among all $H^{1}$-solutions.

Theorem 1.8. Let $p=2$ and $u_{0} \in \mathscr{S}(\mathbb{R}) \backslash\{0\}$ be such that the corresponding solution $u$ of $(K d V)$, which is defined globally in time, is non dispersive for positive times, that is, satisfies (1.6). Then $u$ is a multi-soliton (in $+\infty$ and $-\infty$ ).

The proof of this theorem relies on the soliton resolution result for (KdV), set up by Eckhaus and Schuur [7] and refined in Schuur [30]. 
Remark 1.9. Requiring that the initial condition $u_{0}$ belongs to the Schwartz space is not necessary in order to reach the conclusion in Theorem 1.8. Considering the non dispersion assumption made in Theorem 1.8, it would be sufficient for example that all derivatives up to order 4 of $u_{0}$ decay faster than $x^{-11}$ when $x \rightarrow+\infty$. Actually, we only need to assume that $u_{0}$ is smooth enough and decays sufficiently rapidly for $|x| \rightarrow+\infty$ for the whole of the inverse scattering method to work, thus for the soliton resolution result for $(\mathrm{KdV})$ to hold $[4,30]$. However, our goal is not to obtain the most general statement, and for clarity purposes, we will not attempt to optimize the regularity and decay assumptions on $u_{0}$.

Similarly, we can characterize non dispersive solutions of the (mKdV) equation. Recall that, in addition to solitons, ( $\mathrm{mKdV}$ ) admits other particular solutions, known as breathers, which are also important with respect to the soliton resolution conjecture. Breathers do not correspond to a superposition of solitons but are instead periodic in time and can move both in the left and right directions; for all $(\alpha, \beta) \in \mathbb{R}_{+}^{*} \times \mathbb{R}_{+}^{*}$, and for all $x_{1}, x_{2} \in \mathbb{R}$, the breather $B_{\alpha, \beta, x_{1}, x_{2}}$ with envelope velocity $\gamma:=\beta^{2}-3 \alpha^{2}$, phase velocity $\delta:=3 \beta^{2}-\alpha^{2}$, and translation parameters $x_{1}, x_{2} \in \mathbb{R}$ takes the following expression:

$$
B_{\alpha, \beta, x_{1}, x_{2}}(t, x):=2 \sqrt{2} \partial_{x}\left[\arctan \left(\frac{\beta}{\alpha} \frac{\sin \left(\alpha\left(x-\delta t-x_{1}\right)\right)}{\cosh \left(\beta\left(x-\gamma t-x_{2}\right)\right)}\right)\right] .
$$

We refer to Alejo and Muñoz [1] for the introduction and the study of stability in $H^{2}$ of these solutions. Note that the decomposition result in terms of solitons and breathers available for $(\mathrm{mKdV})$ solutions and stated in [30, Chapter 5, Theorem 5.1] and more recently in [3, Theorem 1.10] holds under the assumption that the initial data $u_{0}$ is generic in the following sense: the set of all $\xi \in \mathbb{C}$ such that the classical Jost solutions $\psi_{l}(\xi)$ and $\psi_{r}(\xi)$ to the Zakharov-Shabat system

$$
\left(\begin{array}{l}
\psi_{1} \\
\psi_{2}
\end{array}\right)^{\prime}=\left(\begin{array}{cc}
-i \xi & u_{0} \\
-u_{0} & i \xi
\end{array}\right)\left(\begin{array}{l}
\psi_{1} \\
\psi_{2}
\end{array}\right)
$$

are $\mathbb{R}$-linearly dependent is finite and consists in the scattering data

$$
\left\{i \sqrt{c_{1}}, \ldots, i \sqrt{c_{N_{1}}}, \alpha_{1}+i \beta_{1}, \ldots, \alpha_{N_{2}}+i \beta_{N_{2}}\right\}
$$

with $N_{1}, N_{2} \in \mathbb{N}, c_{1}, \ldots, c_{N_{1}}, \alpha_{1}, \ldots, \alpha_{N_{2}}, \beta_{1}, \ldots, \beta_{N_{2}} \in \mathbb{R}_{+}^{*}$ such that

$$
c_{1}<\cdots<c_{N}, \quad \beta_{1}^{2}-3 \alpha_{1}^{2}<\cdots<\beta_{N_{2}}^{2}-3 \alpha_{N_{2}}^{2},
$$

and

$$
\forall(i, j) \in\left\{1, \ldots, N_{1}\right\} \times\left\{1, \ldots, N_{2}\right\}, \quad c_{i} \neq \beta_{j}^{2}-3 \alpha_{j}^{2} .
$$

We refer to Schuur [30, Chapter 4], Chen and Liu [3, Paragraph 1.2], and the references therein for more details concerning genericity.

Our result on non dispersive solutions of $(\mathrm{mKdV})$ writes as follows.

Theorem 1.10. Let $p=3$ and $u_{0} \in \mathscr{S}(\mathbb{R}) \backslash\{0\}$ be generic (in the above sense) with scattering data (1.12), and such that the corresponding global solution u of $(m K d V)$ is non dispersive for positive times (that is, satisfies (1.6)).

Then $u$ is a multi-breather with positive speeds in $+\infty$ : we have $N_{1}+N_{2} \geq 1$ and for all $j=1, \ldots, N_{2}$,

$$
\beta_{j}^{2}-3 \alpha_{j}^{2}>0
$$

and there exist $\gamma>0$, positive constants $C_{s}$, signs $\epsilon_{i}= \pm 1$, and real parameters $x_{0, i}, x_{1, j}, x_{2, j}$ such that for all $s \geq 0$, u belongs to $\mathscr{C}\left([0,+\infty), H^{s}(\mathbb{R})\right)$ and

$$
\forall t \geq 0, \quad\left\|u(t)-\sum_{i=1}^{N_{1}} \epsilon_{i} R_{2 c_{i}, x_{0, i}}(t)-\sum_{j=1}^{N_{2}} B_{\sqrt{2} \alpha_{j}, \sqrt{2} \beta_{j}, x_{1, j}, x_{2, j}}(t)\right\|_{H^{s}} \leq C_{s} e^{-\gamma t} .
$$


The proof is done by adapting that of Theorem 1.8 by writing the soliton/breather resolution for $p=3$, and then using smoothness and uniqueness of multi-breathers and the estimates in higher order Sobolev spaces proved by Semenov [31].

Remark 1.11. Let us notice that Remark 1.9 applies also in the context of Theorem 1.10.

1.3. Comments. The proofs of Theorem 1.4 and Corollary 1.6 are in the spirit of the original Liouville result by Martel and Merle [13], and also the work of Laurent and Martel [11] on smoothness and decay of non dispersive solutions. An important ingredient is the observation that a crucial monotonicity formula holds under a much relaxed non dispersion assumption than previously made, see Proposition 2.1, which has its own interest. Also we underline a subtle but key difference in the strategy of the proof: we crucially rely at some point on the asymptotic stability of multi-solitons in the energy space (from [18], stated in Theorem 3.1). But let us recall this result itself is a consequence of the rigidity result for one soliton stated in Theorem 1.1: in some sense, the roles are reversed here.

In fact, our proofs use and combine several previous results on the $(\mathrm{gKdV})$ flow around solitons and multi-solitons. This sheds a new light on many results established so far and which have their own interest, which are here linked together to yield new statements. It seems to us that this phenomenon is an interesting point of this paper.

Our results lead to several open questions. We already mentioned above the first one, but repeat it here: we conjecture that, for each time where defined, multi-solitons for $(\mathrm{gKdV})$ have pointwise exponential decay (along with their derivatives); this is only known in the integrable case, where explicit formulas are known. As second question is whether similar rigidity results (as well as asymptotic stability properties) hold for other dispersive models. The Liouville theorem for solitons holds for the Zakharov-Kuznetsov equation in 2D for example, it would be nice to know if an analog for multi-solitons holds as well. A very natural context is that of the non-linear Schrödinger equations, for which the understanding of nondispersive solutions remains mostly open.

This article is organized as follows. After the introduction, we present in section 2 a general property of exponential decay satisfied by non-dispersive solutions which is an important new observation and interesting in itself. The third section is then devoted to the proof of Theorem 1.4 and Corollary 1.6. In the forth section, we consider the integrable case and sketch the proofs of Theorem 1.8 and 1.10 .

1.4. Acknowledgments. The author would like to thank his supervisor Raphaël Côte for suggesting the idea of this work and for fruitful discussions.

\section{SMOOTHNESS AND EXPONENTIAL DECAY FOR NON DISPERSIVE SOLUTIONS}

The goal of this section is to show the following propositions which extend Laurent and Martel [11, Theorem 1].

Proposition 2.1. Let $J$ be a neighborhood of $+\infty$ and $u \in \mathscr{C}\left(J, H^{1}(\mathbb{R})\right)$ be a solution of $(g K d V)$ which belongs also to $L^{\infty}\left(J, H^{1}(\mathbb{R})\right)$. Suppose that there exists $\rho>0$ such that

$$
\forall \varepsilon>0, \exists R_{\varepsilon}>0, \forall t \in J, \quad \int_{x<\rho t-R_{\varepsilon}} u^{2}(t, x) d x \leq \varepsilon .
$$

Then $u$ belongs to $\mathscr{C}^{\infty}(J \times \mathbb{R})$ and there exists $\kappa>0$ such that for all $k \in \mathbb{N}$, there exists $K_{k}>0$ such that

$$
\forall t \in J, \forall x<\rho t, \quad\left|\partial_{x}^{k} u(t, x)\right| \leq K_{k} e^{-\frac{\kappa}{2}|x-\rho t|} .
$$


We state next another generalized version, which is useful in the proof of Corollary 1.6.

Proposition 2.2. Let $J$ be a neighborhood of $+\infty$ and $u \in \mathscr{C}\left(J, H^{1}(\mathbb{R})\right)$ be a solution of $(g K d V)$ which belongs also to $L^{\infty}\left(J, H^{1}(\mathbb{R})\right)$. Suppose that there exist $\beta, \delta>0$ and two $\mathscr{C}^{1}$ functions $a, b: J \rightarrow \mathbb{R}$ such that

$$
\forall t \in J, \quad \delta \leq a^{\prime}(t) \leq b^{\prime}(t) \leq \beta,
$$

and

$$
\forall \varepsilon>0, \exists R_{\varepsilon}>0, \forall t \in J, \quad \int_{x<m(t)-R_{\varepsilon}} u^{2}(t, x) d x \leq \varepsilon,
$$

where $m(t):=\min \{a(t), b(t)\}$.

Then $u$ belongs to $\mathscr{C}^{\infty}(J \times \mathbb{R})$ and there exists $\kappa>0$ such that for all $k \in \mathbb{N}$, there exists $K_{k}>0$ such that

$$
\forall t \in J, \forall x<m(t), \quad\left|\partial_{x}^{k} u(t, x)\right| \leq K_{k} e^{-\frac{\kappa}{2}|x-m(t)|} .
$$

Remark 2.3. It is to be noticed that, if $J$ in Proposition 2.2 is replaced by a neighborhood $J^{\prime}$ of $-\infty$, then we conclude with an estimate at the right of $M(t):=\max \{a(t), b(t)\}$, or more precisely with the existence of $\kappa>0$ such that for all $k \in \mathbb{N}$, there exists $K_{k}>0$ such that

$$
\forall t \in J^{\prime}, \forall x>M(t), \quad\left|\partial_{x}^{k} u(t, x)\right| \leq K_{k} e^{-\frac{\kappa}{2}(x-M(t))} .
$$

This is justified by the following symmetry property for $(\mathrm{gKdV})$ and the assumption in Propositions 2.2. Denoting $\hat{u}(t, x):=u(-t,-x), \hat{a}(t):=-a(-t), \hat{b}(t):=-b(-t), \hat{m}(t):=-m(-t)$, and $\hat{M}(t):=-M(-t)$, we observe that $u$ satisfies the assumptions of Proposition 2.1 on a neighborhood $J^{\prime}$ of $-\infty$ if and only if $\hat{u}$ satifies the same assumptions on $-J^{\prime}$ (which is a neighborhood of $+\infty$ ) with $a, b, m$, and $M$ replaced respectively by $\hat{a}, \hat{b}, \hat{M}$, and $\hat{m}$ in Proposition 2.2. Thus once we have proved (2.5) as stated in Proposition 2.2, we have immediately the pointwise estimate on $\hat{u}(t)$ at the left of $\hat{m}(t)$ for $t \in-J^{\prime}$, which precisely provides (2.6), that is the desired pointwise estimate on $u(t)$ at the right of $M(t)$ for $t \in J^{\prime}$.

Obviously, Propositions 2.1 and 2.2 apply in particular with $J=\mathbb{R}$, in which case both estimates (2.5) and (2.6) hold.

Now, proceeding essentially as Laurent and Martel [11, Theorem 1], we derive the proof of Proposition 2.2.

\section{Proof. Step 1: Estimates to be established} that

By the classical Sobolev embedding $H^{1}(-\infty, m(t)) \hookrightarrow L^{\infty}(-\infty, m(t))$, it suffices to see

$$
\exists K>0, \forall t \in J, \quad \int_{x<m(t)}\left(u^{2}(t, x)+u_{x}^{2}(t, x)\right) e^{K(m(t)-x)} d x \leq K
$$

holds to have the desired conclusion, that is (2.5), for $k=0$ and for almost every $x<m(t)$. Using that $u(t)$ is continuous on $\mathbb{R}$ by $H^{1}(\mathbb{R}) \hookrightarrow \mathscr{C}(\mathbb{R})$, we deduce that $(2.5)$ is true for $k=0$. Similarly, to reach the whole conclusion, we have to show that for each $k \in \mathbb{N}$, there exists $\tilde{K}_{k}>0$ such that

$$
\forall t \in J, \quad \int_{x<m(t)}\left(\partial_{x}^{k} u(t, x)\right)^{2} e^{\kappa(m(t)-x)} d x \leq \tilde{K}_{k} .
$$

In order to prove (2.8), it is convenient to introduce a well-chosen $\mathscr{C}^{1}$ function defined on $J$, denoted by $\tilde{m}$, which replaces somehow $m$ in the case where $m$ is not already $\mathscr{C}^{1}$. By this means, we get around the difficulty of a possible point where $m$ is not differentiable. This is the purpose of the following:

Claim 2.4. There exists $\tilde{m}: J \rightarrow \mathbb{R}$ of class $\mathscr{C}^{1}$ such that for all $t \in J, m(t) \leq \tilde{m}(t) \leq m(t)+1$, and $\tilde{m}^{\prime}(t) \geq \delta$. 
Proof of Claim 2.4. Define $\tilde{m}$ by

$$
\forall t \in J, \quad \tilde{m}(t):=1+\frac{a(t)+b(t)}{2}-\sqrt{1+\left(\frac{b(t)-a(t)}{2}\right)^{2}} .
$$

Then $\tilde{m}$ is $\mathscr{C}^{1}$ on $J$ and given that $\min \{a, b\}=\frac{a+b}{2}-\frac{|a-b|}{2}$, one can check that $\tilde{m}$ satisfies $m(t) \leq \tilde{m}(t) \leq m(t)+1$ by means of the well-known inequality

$$
\sqrt{x+y} \leq \sqrt{x}+\sqrt{y}, \quad \text { valid for all } x, y \geq 0 .
$$

Moreover, by a straightforward computation, we have

$$
\tilde{m}^{\prime}(t) \geq \frac{a^{\prime}(t)+b^{\prime}(t)}{2}-\frac{b^{\prime}(t)-a^{\prime}(t)}{2} \geq a^{\prime}(t) .
$$

Consequently, Claim 2.4 is proved.

Now, we consider $\tilde{m}$ as in the previous claim. Judging by the fact that for all $t \in J, m(t) \leq$ $\tilde{m}(t)$, we can write for all $k \in \mathbb{N}$

$$
\int_{x<m(t)}\left(\partial_{x}^{k} u(t, x)\right)^{2} e^{\kappa(m(t)-x)} d x \leq \int_{x<\tilde{m}(t)}\left(\partial_{x}^{k} u(t, x)\right)^{2} e^{\kappa(\tilde{m}(t)-x)} d x .
$$

Thus, to achieve our goal (2.8), it suffices to show the existence of $C_{k}>0$ such that

$$
\forall t \in \mathbb{R}, \quad \int_{x<\tilde{m}(t)}\left(\partial_{x}^{k} u(t, x)\right)^{2} e^{\kappa(\tilde{m}(t)-x)} d x \leq C_{k} .
$$

Step 2: Proof of (2.10) for $k=0$

We will obtain (2.10) by a strong monotonicity property which is the purpose of Lemma 2.5 and Lemma 2.6 below.

Let us introduce, for some $\kappa>0$ to be determined later, the function $\varphi$ defined by

$$
\varphi(x)=\frac{1}{2}-\frac{1}{\pi} \arctan \left(e^{\kappa x}\right) .
$$

It satisfies the following properties

$$
\begin{array}{rlrl}
\exists \lambda_{0}>0, \forall x \in \mathbb{R}, & & \lambda_{0} e^{-\kappa|x|} & <-\varphi^{\prime}(x)<\frac{1}{\lambda_{0}} e^{-\kappa|x|}, \\
\forall x \in \mathbb{R}, & \left|\varphi^{(3)}(x)\right| & \leq-\kappa^{2} \varphi^{\prime}(x) . \\
\exists \lambda_{1}>0, \forall x \geq 0, & \lambda_{1} e^{-\kappa x} & \leq \varphi(x) .
\end{array}
$$

Moreover, let us observe that

$$
\int_{x<\tilde{m}(t)} u^{2}(t, x) e^{\kappa(\tilde{m}(t)-x)} d x=\int_{x<0} u^{2}(t, x+\tilde{m}(t)) e^{-\kappa x} d x,
$$

and that, for all $x_{0}<0$,

$$
\begin{aligned}
\int_{x_{0} \leq x<0} u^{2}(t, x+\tilde{m}(t)) e^{-\kappa x} d x & \leq e^{-\kappa x_{0}} \int_{x \geq x_{0}} u^{2}(t, x+\tilde{m}(t)) e^{-\kappa\left(x-x_{0}\right)} d x \\
& \leq \frac{1}{\lambda_{1}} e^{-\kappa x_{0}} \int_{x \geq x_{0}} u^{2}(t, x+\tilde{m}(t)) \varphi\left(x-x_{0}\right) d x \\
& \leq \frac{1}{\lambda_{1}} e^{-\kappa x_{0}} \int_{\mathbb{R}} u^{2}(t, x+\tilde{m}(t)) \varphi\left(x-x_{0}\right) d x .
\end{aligned}
$$

By Claim 2.4, for all $t \in J, \tilde{m}^{\prime}(t) \geq \delta$. Therefore there exists $\eta>0$ and an increasing affine function $f: \mathbb{R} \rightarrow \mathbb{R}$ such that

$$
\forall t \in J, \quad-f^{\prime}(t)+\tilde{m}^{\prime}(t) \geq \eta
$$


Now, for fixed $t_{0} \in J$ and $x_{0}$ in $\mathbb{R}$, consider

$$
\begin{aligned}
I_{\left(t_{0}, x_{0}\right)}: \mathbb{R} & \rightarrow \mathbb{R}^{+} \\
t & \mapsto \int_{\mathbb{R}} u^{2}(t, x+\tilde{m}(t)) \varphi\left(x-x_{0}+f(t)-f\left(t_{0}\right)\right) d x
\end{aligned}
$$

We have

$$
\forall t \in \mathbb{R}, \quad I_{\left(t_{0}, x_{0}\right)}(t)=\int_{\mathbb{R}} u^{2}(t, x) \varphi\left(x-x_{0}+f(t)-f\left(t_{0}\right)-\tilde{m}(t)\right) d x,
$$

so that by derivation with respect to $t$, we obtain

$$
\begin{aligned}
\frac{d I_{\left(t_{0}, x_{0}\right)}}{d t}(t)= & -3 \int_{\mathbb{R}} u_{x}^{2}(t, x) \varphi^{\prime}(\tilde{x}) d x-\left(-f^{\prime}(t)+\tilde{m}^{\prime}(t)\right) \int_{\mathbb{R}} u^{2}(t, x) \varphi^{\prime}(\tilde{x}) d x \\
& +\int_{\mathbb{R}} u^{2}(t, x) \varphi^{(3)}(\tilde{x}) d x+\frac{2 p}{p+1} \int_{\mathbb{R}} u^{p+1}(t, x) \varphi^{\prime}(\tilde{x}) d x,
\end{aligned}
$$

where $\tilde{x}:=x-x_{0}+f(t)-f\left(t_{0}\right)-\tilde{m}(t)$.

Set $\kappa:=\sqrt{\frac{\eta}{2}}$. We claim then

Lemma 2.5. There exists $C_{0}>0$ such that for all $x_{0} \in \mathbb{R}$, and for all $t_{0}, t \in J$,

$$
\frac{d I_{\left(t_{0}, x_{0}\right)}}{d t}(t) \geq-C_{0} e^{-\kappa\left(-x_{0}+f(t)-f\left(t_{0}\right)\right)} .
$$

Proof of Lemma 2.5. Due to the choice of $\kappa$ and property (2.12) of $\varphi$, we have

$$
\left|\int_{\mathbb{R}} u^{2}(t, x) \varphi^{(3)}(\tilde{x}) d x\right| \leq-\frac{\eta}{2} \int_{\mathbb{R}} u^{2}(t, x) \varphi^{\prime}(\tilde{x}) d x .
$$

Furthermore we control the non-linear part by considering, for $R>0$,

and

$$
I_{1}(t):=\int_{|\tilde{x}|>-x_{0}-R+f(t)-f\left(t_{0}\right)} u^{p+1}(t, x) \varphi^{\prime}(\tilde{x}) d x
$$

On the one hand, we have due to (2.11)

$$
\left|I_{1}(t)\right| \leq \frac{1}{\lambda_{0}} e^{-\kappa\left(-x_{0}-R+f(t)-f\left(t_{0}\right)\right)}\left(\int_{\mathbb{R}}|u|^{p+1}(t, x) d x\right) \leq C e^{-\kappa\left(-x_{0}-R+f(t)-f\left(t_{0}\right)\right)},
$$

where we have used the Sobolev embedding $H^{1}(\mathbb{R}) \hookrightarrow L^{p+1}(\mathbb{R})$ and the fact that $u$ belongs to $L^{\infty}\left(J, H^{1}(\mathbb{R})\right)$. Note that $C>0$ is independent of $x_{0}, t_{0}$, and $t$.

On the other, we observe that if $|\tilde{x}| \leq-x_{0}-R+f(t)-f\left(t_{0}\right)$, then $x \leq \tilde{m}(t)-R$ in particular, and therefore by Claim 2.4 we have also $x \leq m(t)-R+1$. Thus, it follows

$$
\begin{aligned}
\left|I_{2}(t)\right| & \leq\|u(t)\|_{L^{\infty}(x \leq m(t)-R+1)}^{p-1} \int_{x \leq m(t)-R+1} u^{2}(t, x)\left|\varphi^{\prime}(x)\right| d x \\
& \leq \sqrt{2}\|u(t)\|_{L^{2}(x \leq m(t)-R+1)}^{\frac{p-1}{2}}\left\|u_{x}(t)\right\|_{L^{2}(x \leq m(t)-R+1)}^{\frac{p-1}{2}} \int_{\mathbb{R}} u^{2}(t, x)\left|\varphi^{\prime}(x)\right| d x \\
& \leq \sqrt{2}\|u(t)\|_{L^{2}(x \leq m(t)-R+1)}^{\frac{p-1}{2}} \sup _{t \in \mathbb{R}}\|u(t)\|_{H^{1}}^{\frac{p-1}{2}} \int_{\mathbb{R}} u^{2}(t, x)\left|\varphi^{\prime}(x)\right| d x .
\end{aligned}
$$

By the non-dispersion assumption (2.1), we can choose $R>1$ such that

$$
\sqrt{2}\|u(t)\|_{L^{2}(x \leq m(t)-R+1)}^{\frac{p-1}{2}} \sup _{t \in \mathbb{R}}\|u(t)\|_{H^{1}}^{\frac{p-1}{2}} \leq \frac{p+1}{4 p} \eta .
$$

Taking into account (2.21), this leads eventually to the following estimate

$$
\frac{2 p}{p+1}\left|\int_{\mathbb{R}} u^{p+1}(t, x) \varphi^{\prime}(\tilde{x}) d x\right| \leq-\frac{\eta}{2} \int_{\mathbb{R}} u^{2}(t, x) \varphi^{\prime}(x) d x+C_{0} e^{-\kappa\left(-x_{0}-R+f(t)-f\left(t_{0}\right)\right)},
$$


where $C_{0}:=\frac{2 p}{p+1} C$ is independent of $x_{0}, t_{0}$, and $t$. Gathering (2.16), (2.20), and (2.22) in (2.18) we deduce finally

$$
\frac{d I_{\left(t_{0}, x_{0}\right)}}{d t}(t) \geq-3 \int_{\mathbb{R}} u_{x}^{2}(t, x) \varphi^{\prime}(\tilde{x}) d x-C_{0} e^{-\kappa\left(-x_{0}-R+f(t)-f\left(t_{0}\right)\right)} .
$$

Thus Lemma 2.5 is established.

As a consequence of the preceding lemma,

$$
\exists C_{1}>0, \forall x_{0} \in \mathbb{R}, \forall t \geq t_{0}, \quad I_{\left(t_{0}, x_{0}\right)}\left(t_{0}\right) \leq I_{\left(t_{0}, x_{0}\right)}(t)+C_{1} e^{\kappa x_{0}},
$$

with $C_{1}$ independent of the parameters $x_{0}$ and $t_{0}$. Next, we claim the following:

Lemma 2.6. For fixed $x_{0} \in \mathbb{R}$ and $t_{0} \in J, I_{\left(t_{0}, x_{0}\right)}(t) \rightarrow 0$ as $t \rightarrow+\infty$.

Proof. To show this lemma, we just repeat the arguments given by Laurent and Martel [11, paragraph 2.1, Step 2]. Let $\varepsilon$ be a positive real number. By Claim 2.4 and by (2.4), there exists $\tilde{R}>0$ such that

$$
\int_{x<\tilde{m}(t)-\tilde{R}} u^{2}(t, x) d x \leq \frac{\varepsilon}{2} .
$$

Since $0 \leq \varphi \leq 1$, this enables us to see that

$$
\int_{x<-\tilde{R}} u^{2}(t, x+\tilde{m}(t)) \varphi\left(x-x_{0}+f(t)-f\left(t_{0}\right)\right) d x \leq \int_{x<\tilde{m}(t)-\tilde{R}} u^{2}(t, x) d x \leq \frac{\varepsilon}{2} .
$$

Now, recall that $\varphi$ is decreasing so that

$$
\begin{gathered}
\int_{x \geq-\tilde{R}} u^{2}(t, x+\tilde{m}(t)) \varphi\left(x-x_{0}+f(t)-f\left(t_{0}\right)\right) d x \\
\leq \varphi\left(-\tilde{R}-x_{0}+f(t)-f\left(t_{0}\right)\right)\|u(t)\|_{L^{2}}^{2} \\
\leq \bar{C} \varphi\left(-\tilde{R}-x_{0}+f(t)-f\left(t_{0}\right)\right),
\end{gathered}
$$

with $\bar{C}=\|u(t)\|_{L^{2}}^{2}$ for all $t \in J$. Moreover, since $f(t) \rightarrow+\infty$ as $t \rightarrow+\infty$ and $\varphi(x) \rightarrow 0$ as $x \rightarrow+\infty$, there exists $T \in \mathbb{R}$ such that for all $t \geq T$,

$$
\bar{C} \varphi\left(-\tilde{R}-x_{0}+f(t)-f\left(t_{0}\right)\right) \leq \frac{\varepsilon}{2} .
$$

Then, for all $t \geq T$,

$$
I_{\left(t_{0}, x_{0}\right)}(t) \leq \frac{\epsilon}{2}+\frac{\varepsilon}{2}=\varepsilon .
$$

Hence, we have finished proving Lemma 2.6.

Due to (2.23) and Lemma 2.6, we obtain

$$
\forall t_{0} \in J, \forall x_{0} \in \mathbb{R}, \quad I_{\left(t_{0}, x_{0}\right)}\left(t_{0}\right) \leq C_{1} e^{\kappa x_{0}} .
$$

Thus, (2.15) leads to: for all $t \in J$,

$$
\int_{x_{0} \leq x<0} u^{2}(t, x+\tilde{m}(t)) e^{-\kappa x} d x \leq \frac{C_{1}}{\lambda_{1}} .
$$

Thus letting $x_{0}$ tend to $-\infty$, we deduce from (2.14) that

$$
\int_{x<\tilde{m}(t)} u^{2}(t, x) e^{\kappa(\tilde{m}(t)-x)} d x \leq \frac{C_{1}}{\lambda_{1}} .
$$

Step 3: Proof of (2.8) for $k \geq 1$

Starting from the fact that for all $t \geq t_{0}$ and for all $x_{0}<0$

$$
I_{\left(t_{0}, x_{0}\right)}\left(t_{0}\right)-I_{\left(t_{0}, x_{0}\right)}(t) \leq \frac{C_{1}}{\lambda_{1}} e^{\kappa x_{0}}+3 \int_{t_{0}}^{t} \int_{\mathbb{R}} u_{x}^{2}(s, x+\tilde{m}(s)) \varphi^{\prime}\left(x-x_{0}+f(s)-f\left(t_{0}\right)\right) d x d s
$$


and arguing like Laurent and Martel [11, paragraph 2.1 Step 3], one can show

$$
\int_{t_{0}}^{t_{0}+1} \int_{\mathbb{R}} u_{x}^{2}(s, x+\tilde{m}(s)) e^{\kappa x} d x d s \leq C,
$$

where $C$ is independent of $x_{0}$ and $t_{0}$.

Now, one proves by induction on $k \in \mathbb{N}$ the existence of $C_{k} \geq 0$ such that for all $t \in \mathbb{R}$, $\|u(t)\|_{H^{k}} \leq C_{k}$ and

$$
\int_{\mathbb{R}}\left(\partial_{x}^{k} u\right)^{2}(t, x+\tilde{m}(t)) e^{\kappa x} d x+\int_{t}^{t+1} \int_{\mathbb{R}}\left(\partial_{x}^{k} u\right)^{2}(s, x+\tilde{m}(s)) e^{\kappa x} d x d s \leq C_{k} .
$$

In particular, estimates (2.8) are then performed. Moreover, we deduce from the equation satisfied by $u$ that the partial derivatives with respect to $x$ and $t$ of all order exist and are continuous, thus $u \in \mathscr{C}^{\infty}(J \times \mathbb{R})$.

For simplification purposes, we will not explicit the proof of (2.27) and refer instead to [11, paragraph 2.3 and paragraph 2.2 Step 2]; the induction argument works since we assume $b^{\prime}(t) \leq \beta$ in (2.3), which implies that $\tilde{m}$ is bounded on $J$.

\section{Proof of Theorem 1.4 and Corollary 1.6}

We split the proof into four steps. The three first steps are common to both theorems and are valid under the hypotheses of Theorem 1.4 , whereas the last one is specific to the proof of Corollary 1.6 where exponential decay properties are established and for which the stronger non dispersion assumption (1.9) is required.

Consider $u$ which satisfies the assumptions of Theorem 1.4.

Step 1: Asymptotic stability in the energy space

The following asymptotic stability result in the energy space is to be considered as a crucial tool for the proof.

Theorem 3.1 (Martel, Merle and Tsai [26]; Martel and Merle [19]). Fix $0<c_{1}^{0}<\cdots<c_{N}^{0}$. For all $\beta>0$, there exist $L_{0}>0$ and $\alpha_{0}=\alpha_{0}(\beta)>0$ such that if $u \in \mathscr{C}\left([0,+\infty), H^{1}(\mathbb{R})\right)$ is a solution of $(g K d V)$ satisfying

$$
\forall t \geq 0, \quad \inf _{\substack{r_{i} \in \mathbb{R} \\ r_{i+1}-r_{i}>L_{0}}}\left\|u(t)-\sum_{i=1}^{N} Q_{c_{i}^{0}}\left(\cdot-r_{i}\right)\right\|_{H^{1}}<C\left(\alpha_{0}+e^{-\gamma t}\right)
$$

for some positive constants $C$ and $\gamma$, then the following holds.

(1) (Asymptotic stability in the energy space) There exist $\mathscr{C}^{1}$ functions $t \mapsto c_{i}(t) \in \mathbb{R}_{+}^{*}$, $t \mapsto \rho_{i}(t) \in \mathbb{R}$ for $i \in\{1, \ldots, N\}$ such that

$$
\lim _{t \rightarrow+\infty}\left\|u(t)-\sum_{i=1}^{N} Q_{c_{i}(t)}\left(\cdot-\rho_{i}(t)\right)\right\|_{H^{1}(x>\beta t)}=0 .
$$

(2) (Convergence of the scaling parameter) There exists $c_{i}^{+} \in \mathbb{R}_{+}^{*}$ such that $\lim _{t \rightarrow+\infty} c_{i}(t)=$ $c_{i}^{+}$.

Set $\delta:=\frac{1}{2} \min \left\{c_{1}, \min _{i \in\{1, \ldots, N-1\}}\left\{c_{i+1}-c_{i}\right\}\right\}$.

Using Theorem 3.1 and adapting the classical modulation argument set up in the proof by 
Martel and Merle [19, section 5], we have the following. For all $i \in\{1, \ldots, N\}$, there exist $c_{i}:[0,+\infty) \rightarrow \mathbb{R}_{+}^{*}, \rho_{i}:[0,+\infty) \rightarrow \mathbb{R}$ of class $\mathscr{C}^{1}$ such that, defining

$$
\epsilon:(t, x) \mapsto u(t, x)-\sum_{i=1}^{N} Q_{c_{i}(t)}\left(x-\rho_{i}(t)\right),
$$

and for $\alpha$ small enough in Theorems 1.4 and 1.6, we have

(1) the conclusion of Theorem 3.1, that is

$$
\lim _{t \rightarrow+\infty}\|\epsilon(t)\|_{H^{1}\left(x>\frac{c_{1}}{A} t\right)}=0
$$

with $A>3$ such that

$$
\frac{c_{1}}{A}<\rho-\sigma,
$$

(take $\sigma=0$ for Theorem 1.4) and

$$
\forall i \in\{1, \ldots, N\}, \exists c_{i}^{+} \in \mathbb{R}_{+}^{*}, \quad \lim _{t \rightarrow+\infty} c_{i}(t)=c_{i}^{+} ;
$$

(2) control on the modulation parameters [26, proof of Lemma 1]: more precisely, there exists $K>0$ such that for all $t$ large enough,

$$
\forall i \in\{1, \ldots, N-1\}, \quad \rho_{i+1}(t)-\rho_{i}(t) \geq \delta t,
$$

and for all $i \in\{1, \ldots, N\}$,

$$
\begin{gathered}
\left|c_{i}(t)-c_{i}\right|+\|\epsilon(t)\|_{H^{1}} \leq \frac{\delta}{K+1}, \\
\left|\rho_{i}^{\prime}(t)-c_{i}(t)\right| \leq K\left(\int_{\mathbb{R}} \epsilon^{2}(t, x) e^{-\sqrt{c_{1}}\left|x-\rho_{i}(t)\right|} d x\right)^{\frac{1}{2}} .
\end{gathered}
$$

Remark 3.2. The preceding choices of $A, K$, and of the functions $t \mapsto c_{i}(t)$ as defined before are possible, provided $\alpha$ is sufficiently small.

Note also that estimate (3.8) and assertion (3.6) guarantee that $0<c_{1}^{+}<\cdots<c_{N}^{+}$(due to the choice of $\delta$ ).

Step 2: Convergence of $u(t)-\sum_{i=1}^{N} Q_{c_{i}^{+}}\left(\cdot-\rho_{i}(t)\right)$ as $t \rightarrow+\infty$

Lemma 3.3. We have

$$
\left\|u(t)-\sum_{i=1}^{N} Q_{c_{i}^{+}}\left(\cdot-\rho_{i}(t)\right)\right\|_{H^{1}} \rightarrow 0, \quad \text { as } t \rightarrow+\infty .
$$

This lemma follows immediately from Claim 3.4 and Claim 3.5 below. We begin with this first observation.

Claim 3.4. We have

$$
\lim _{t \rightarrow+\infty}\left\|u(t)-\sum_{i=1}^{N} Q_{c_{i}^{+}}\left(\cdot-\rho_{i}(t)\right)\right\|_{H^{1}\left(x>\frac{c_{1}}{A} t\right)}=0 .
$$

Let us justify this fact.

Using the triangular inequality and taking into account (3.4), it suffices in fact to see that for all $i \in\{1, \ldots, N\}$,

$$
\lim _{t \rightarrow+\infty}\left\|Q_{c_{i}(t)}\left(\cdot-\rho_{i}(t)\right)-Q_{c_{i}^{+}}\left(\cdot-\rho_{i}(t)\right)\right\|_{H^{1}\left(x>\frac{c_{1}}{A} t\right)}=0 .
$$


But the quantity $\left\|Q_{c_{i}(t)}\left(\cdot-\rho_{i}(t)\right)-Q_{c_{i}^{+}}\left(\cdot-\rho_{i}(t)\right)\right\|_{H^{1}\left(x>\frac{c_{1}}{A} t\right)}$ is bounded by $\left\|Q_{c_{i}(t)}-Q_{c_{i}^{+}}\right\|_{H^{1}}$ which tends to 0 as $t$ tends to $+\infty$. We recall indeed that the map

$$
\mathbb{R}_{+}^{*} \rightarrow H^{1}(\mathbb{R}), \quad c \mapsto Q_{c}
$$

is continuous by application of Lebesgue's dominated convergence theorem. Hence (3.11) holds and Claim 3.4 is proved.

Due to the assumption of non-dispersion made in Theorem 1.4, we claim moreover:

Claim 3.5. We have

$$
\lim _{t \rightarrow+\infty}\left\|u(t)-\sum_{i=1}^{N} Q_{c_{i}^{+}}\left(\cdot-\rho_{i}(t)\right)\right\|_{H^{1}\left(x \leq \frac{c_{1}}{A} t\right)}=0 .
$$

In what follows, we prove actually that each quantity $\|u(t)\|_{H^{1}\left(x \leq \frac{c_{1}}{A} t\right)}$ and $\left\|Q_{c_{i}^{+}}\left(\cdot-\rho_{i}(t)\right)\right\|_{H^{1}\left(x \leq \frac{c_{1}}{A} t\right)}$ for $i \in\{1, \ldots, N\}$ tends to 0 as $t$ tends to $+\infty$.

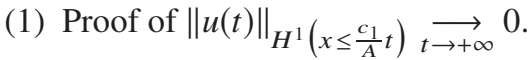

Let $\varepsilon>0$. By (1.6) or (1.9), there exists $R_{\varepsilon}>0$ such that for all $R \geq R_{\varepsilon}$,

$$
\forall t \geq 0, \quad \int_{x<(\rho-\sigma) t-R} u^{2}(t, x) d x \leq \frac{\varepsilon}{2} .
$$

Now, by means of Proposition 2.1, there exist $\kappa>0$ and $K_{1}>0$ such that for all $t \geq 0$,

$$
\forall x \leq(\rho-\sigma) t, \quad\left|u_{x}(t, x)\right| \leq K_{1} e^{-\kappa|x-(\rho-\sigma) t|} .
$$

Pick $R \geq R_{\epsilon}$ such that $K_{1}^{2} e^{-\kappa R} \int_{\mathbb{R}} e^{-\kappa|x|} d x \leq \frac{\varepsilon}{2}$.

For $t$ large enough, $\frac{c_{1}}{A} t<(\rho-\sigma) t-R$ due to (3.5), and therefore

$$
\int_{x \leq \frac{c_{1}}{A} t} u^{2}(t, x) d x \leq \frac{\varepsilon}{2}
$$

and

$$
\begin{aligned}
\int_{x \leq \frac{c_{1}}{A} t} u_{x}^{2}(t, x) d x & \leq \int_{x \leq(\rho-\sigma) t-R} u_{x}^{2}(t, x) d x \leq K_{1} \int_{x \leq(\rho-\sigma) t-R} e^{-\kappa R}\left|u_{x}(t, x)\right| d x \\
& \leq K_{1}^{2} e^{-\kappa R} \int_{x \leq(\rho-\sigma) t-R} e^{-\kappa|x-(\rho-\sigma) t|} d x \\
& \leq K_{1}^{2} e^{-\kappa R} \int_{\mathbb{R}} e^{-\kappa|x-(\rho-\sigma) t|} d x \leq \frac{\varepsilon}{2}
\end{aligned}
$$

As a consequence, for $t$ large enough, $\|u(t)\|_{H^{1}\left(x \leq \frac{c_{1}}{A} t\right)}^{2} \leq \varepsilon$.

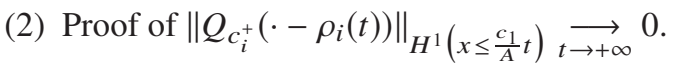

Notice first that, recalling (3.8) and (3.9), we have for $t$ large enough

$$
\begin{aligned}
\left|\rho_{i}^{\prime}(t)-c_{i}\right| & \leq\left|\rho_{i}^{\prime}(t)-c_{i}(t)\right|+\left|c_{i}(t)-c_{i}\right| \leq K\|\epsilon(t)\|_{L^{2}}+\left|c_{i}(t)-c_{i}\right| \\
& \leq(K+1)\left(\|\epsilon(t)\|_{L^{2}}+\left|c_{i}(t)-c_{i}\right|\right) \leq \frac{c_{1}}{2} .
\end{aligned}
$$

In particular, for $t$ large enough,

$$
\rho_{i}^{\prime}(t) \geq \frac{c_{1}}{2}
$$


By integration of the preceding inequality, we deduce that for large values of $t, \rho_{i}(t) \geq$ $\frac{c_{1}}{3} t$. Thus, for these values,

$$
\rho_{i}(t)-\frac{c_{1}}{A} t \geq c_{1}\left(\frac{1}{3}-\frac{1}{A}\right) t
$$

with $\frac{1}{3}-\frac{1}{A}>0$.

Due to the exponential decay property of the integrable functions $Q_{c_{i}^{+}}$and $Q_{c_{i}^{+}}^{\prime}$, we

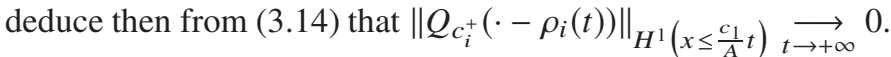

Now, it follows from Claim 3.4 and Claim 3.5 that

$$
\lim _{t \rightarrow+\infty}\left\|u(t)-\sum_{i=1}^{N} Q_{c_{i}^{+}}\left(\cdot-\rho_{i}(t)\right)\right\|_{H^{1}}=0 .
$$

Step 3: Refinement of (3.15)

Proposition 3.6 (Improvement of the $H^{1}$-convergence for asymptotic $N$-soliton like solutions). Let $u \in \mathscr{C}\left(\mathbb{R}, H^{1}(\mathbb{R})\right)$ be a solution of $(g K d V)$ and let $0<c_{1}<\cdots<c_{N}$. Assume the existence of $T_{0} \geq 0, \delta_{0}>0$, and $N$ functions $x_{1}, \ldots, x_{N}: \mathbb{R} \rightarrow \mathbb{R}$ of class $\mathscr{C}^{1}$ satisfying for all $t \geq T_{0}$,

(3.16) $\forall i=1, \ldots, N-1, \quad x_{i+1}(t)-x_{i}(t) \geq \delta_{0} t \quad$ and $\quad \forall i=1, \ldots, N, \quad x_{i}^{\prime}(t) \geq \delta_{0}$, and such that

$$
\lim _{t \rightarrow+\infty}\left\|u(t)-\sum_{i=1}^{N} Q_{c_{i}}\left(\cdot-x_{i}(t)\right)\right\|_{H^{1}}=0 .
$$

Then there exist $C>0$ and $y_{1}, \ldots, y_{N} \in \mathbb{R}$ such that

$$
\forall t \geq 0, \quad\left\|u(t)-\sum_{i=1}^{N} Q_{c_{i}}\left(\cdot-c_{i} t-y_{i}\right)\right\|_{H^{1}} \leq C e^{-\frac{1}{8} \delta_{0}^{\frac{3}{2}} t} .
$$

It was first observed by Martel [12, Proposition 4] that multi-solitons in the sense of Definition 1.2 do actually converge exponentially fast to their profile: this was a key to proving uniqueness of multi-solitons, in the $L^{2}$-subcritical case. In the above Proposition 3.6 we further refine this observation, by noticing that the conclusion still holds even if one gives some freedom to the center of mass of the soliton $x_{i}(t)$ (instead of (3.16), the assumption in [12, Proposition 4] was $\left.x_{i}(t)=c_{i} t+y_{i}\right)$.

The proof of Proposition 3.6 follows the lines of [12] and is postponed to the Appendix; we go on assuming it holds.

Given (3.7), (3.13), and (3.15), we just have to apply the previous proposition (with $x_{i}$ replaced by $\rho_{i}$ and $\delta_{0}$ by $\delta$ defined in Step 1 ) to conclude that $u$ is a multi-soliton. In other words, there exist $x_{1}^{+}, \ldots, x_{N}^{+} \in \mathbb{R}$ such that

$$
\forall t \geq 0, \quad\left\|u(t)-\sum_{i=1}^{N} Q_{c_{i}^{+}}\left(\cdot-c_{i}^{+} t-x_{i}^{+}\right)\right\|_{H^{1}} \leq C e^{-\frac{1}{8} \delta^{\frac{3}{2}} t} .
$$

We recall then from Martel [12, proof of Proposition 5] that for all $s \in \mathbb{N}^{*}, u \in \mathscr{C}\left([0,+\infty), H^{s}(\mathbb{R})\right)$ and there exists $\tilde{C}_{S} \geq 0$ such that

$$
\forall t \geq 0, \quad\left\|u(t)-\sum_{i=1}^{N} Q_{c_{i}^{+}}\left(\cdot-c_{i}^{+} t-x_{i}^{+}\right)\right\|_{H^{s}} \leq \tilde{C}_{s} e^{-\frac{1}{32} \delta^{\frac{3}{2}} t} .
$$

This concludes the proof of Theorem 1.4. 
Step 4: Proof of smoothness and exponential decay of $u$

Apply Proposition 2.2 with $a(t):=(\rho-\sigma) t$ and $b(t):=(\rho+\sigma) t$ to obtain $u \in \mathscr{C}^{\infty}([0,+\infty) \times \mathbb{R})$ and for each $t \geq 0$,

$$
\forall x \leq(\rho-\sigma) t, \quad\left|\partial_{x}^{s} u(t, x)\right| \leq K_{s} e^{-\gamma|x-(\rho-\sigma) t|},
$$

where $K_{s}, \gamma>0$ are independent of $t$ and $x$.

Under the global non-dispersion assumption of Corollary 1.6, which we take as granted from now on, we have also

$$
\forall x \geq(\rho+\sigma) t, \quad\left|\partial_{x}^{s} u(t, x)\right| \leq K_{s} e^{-\gamma(x-(\rho+\sigma) t)} .
$$

(See Remark 2.3.)

At this stage and as we explain just below, the desired exponential decay estimate (1.10) follows from the strong property (1.5) or (3.20). We distinguish three cases, depending on the position of $x$ with respect to $\pm 2(\rho+\sigma) t$; the moral being that (1.5) implies the expected pointwise estimate in each region $|x| \leq \zeta t$ (with an exponential decay rate depending on $\zeta$ ) and even if it means taking $\zeta$ large enough and reducing the decay rate $\gamma$, one can propagate the control by $e^{-\gamma\left|x-c_{N}^{+} t\right|}$ (respectively $e^{-\gamma\left|x-c_{1}^{+} t\right|}$ ) to the region $x>(\rho+\sigma) t$ (respectively $x<(\rho-\sigma) t)$.

Let $t \geq 0$ and $\tilde{\theta}:=\frac{1}{32} \delta^{\frac{3}{2}}$, for each $s \in \mathbb{N}$, there exists $\tilde{K}_{s}>0$ such that

$$
\left\|\partial_{x}^{s}\left(u(t)-\sum_{i=1}^{N} Q_{c_{i}^{+}}\left(\cdot-c_{i}^{+} t-x_{i}^{+}\right)\right)\right\|_{L^{\infty}} \leq \tilde{K}_{s} e^{-\tilde{\theta} t} .
$$

Case 1: $|x| \leq 2(\rho+\sigma) t$. We have

$$
\left|x-c_{N}^{+} t\right| \leq\left(2(\rho+\sigma)+c_{N}^{+}\right) t \quad \text { that is, } \frac{\tilde{\theta}}{2(\rho+\sigma)+c_{N}^{+}}\left|x-c_{N}^{+} t\right| \leq \tilde{\theta} t,
$$

and thus

$$
\left\|\partial_{x}^{s}\left(u(t)-\sum_{i=1}^{N} Q_{c_{i}^{+}}\left(\cdot-c_{i}^{+} t-x_{i}^{+}\right)\right)\right\|_{L^{\infty}} \leq \tilde{K}_{s} e^{-\frac{\tilde{\theta}}{2(\rho+\sigma)+c_{N}^{+}}\left|x-c_{N}^{+} t\right|} .
$$

Consequently for $t \geq 0$ and $|x| \leq 2(\rho+\sigma) t$, using the triangular inequality and the exponential decay of $\partial_{x}^{s} Q_{c_{i}^{+}}$, we obtain

$$
\begin{aligned}
\left|\partial_{x}^{s} u(t, x)\right| & \leq \sum_{i=1}^{N}\left|\partial_{x}^{s} Q_{c_{i}^{+}}\left(x-c_{i}^{+} t-x_{i}^{+}\right)\right|+\left\|\partial_{x}^{s}\left(u(t)-\sum_{i=1}^{N} Q_{c_{i}^{+}}\left(\cdot-c_{i}^{+} t-x_{i}^{+}\right)\right)\right\|_{L^{\infty}} \\
& \leq \tilde{\tilde{K}}_{s} \sum_{i=1}^{N} e^{-\tilde{\gamma}\left|x-c_{i}^{+} t\right|}
\end{aligned}
$$

where $\tilde{\gamma}:=\min \left\{\sqrt{c_{1}^{+}}, \frac{\tilde{\theta}}{2(\rho+\sigma)+c_{N}^{+}}\right\}$.

Case 2: $x \geq 2(\rho+\sigma) t$. Let us rewrite this as $x-(\rho+\sigma) t \geq \frac{1}{2} x$. In particular

$$
x-(\rho+\sigma) t \geq \frac{1}{2}\left(x-c_{N}^{+} t\right)
$$

so that for $x \geq 2(\rho+\sigma) t$,

$$
\left|\partial_{x}^{s} u(t, x)\right| \leq K_{s} e^{-\gamma(x-(\rho+\sigma) t)} \leq K_{s} e^{-\frac{\gamma}{2}\left(x-c_{N}^{+} t\right)} .
$$

Case 3: $x \leq-2(\rho+\sigma) t$. Arguing similarly as before, we have then

$$
\left|\partial_{x}^{s} u(t, x)\right| \leq K_{s} e^{-\gamma((\rho-\sigma) t-x)} \leq K_{s} e^{-\frac{\gamma}{L}\left(c_{1}^{+} t-x\right)},
$$


for $L>2$ chosen such that $\frac{L(\rho-\sigma)-c_{1}^{+}}{L-1}>-2(\rho+\sigma)$.

Set finally $\theta:=\min \left\{\frac{\gamma}{L}, \tilde{\gamma}\right\}$ to obtain (1.10) in Corollary 1.6.

\section{The integrable Cases: Proofs of Theorems 1.8 and 1.10}

4.1. Non dispersive solutions of the Korteweg-de Vries equation. The strategy to prove Theorem 1.8 takes inspiration in [11, Proof of Theorem 2]. We use the following result of Eckhaus and Schuur [7, Section 5], which is also a consequence of a generalized version by Schuur [30, Chapter 2, Theorem 7.1 and (7.23)].

Theorem 4.1 (Eckhaus and Schuur [7]; Schuur [30]). Let $p=2$ and $u_{0} \in \mathscr{C}^{4}(\mathbb{R})$ be such that for some $C_{0}>0$, for all $k=0, \ldots, 4$, and for all $x \in \mathbb{R}$,

$$
\left|\frac{\partial^{k} u_{0}(x)}{\partial x^{k}}\right| \leq C_{0}|x|^{-11} .
$$

Let $u$ be the corresponding global solution of $(K d V)$.

Then there exists a solution $u_{d}$ which is a multi-soliton or zero such that for all $\beta>0$, there exist $\gamma=\gamma(\beta)>0$ and $K \geq 0$ such that for all $t>0$

$$
\left\|u(t)-u_{d}(t)\right\|_{L^{\infty}(x>\beta t)}+\left\|u(t)-u_{d}(t)\right\|_{L^{2}(x>\beta t)} \leq K t^{-\frac{1}{3}} .
$$

Proof of Theorem 1.8. Due to the assumption on $u_{0}$ in Theorem 1.8, we can apply Theorem 4.1 and we obtain a solution $u_{d}$ of $(\mathrm{KdV})$ as above which fulfills (4.2). We claim first that $u_{d}$ is not the trivial solution. Otherwise, with $\beta:=\frac{\rho}{2}>0$, we would have $\|u(t)\|_{L^{2}(x>\beta t)}=$ $\mathrm{O}\left(t^{-\frac{1}{3}}\right)$ as $t$ tends to $+\infty$. On the other hand, by the non dispersion assumption and namely by Proposition 2.1,

$$
\forall x \leq \beta t, \quad|u(t, x)| \leq C e^{-\gamma|x-\rho t|},
$$

so that

$$
\|u(t)\|_{L^{2}(x \leq \beta t)} \leq C e^{-\gamma \beta t} .
$$

Then, we would obtain that

$$
\|u(t)\|_{L^{2}}=\|u(t)\|_{L^{2}(x \leq \beta t)}+\|u(t)\|_{L^{2}(x>\beta t)} \rightarrow 0 \quad \text { as } t \rightarrow+\infty,
$$

hence conclude that $\left\|u_{0}\right\|_{L^{2}}=0$ by the mass conservation law. This contradicts our assumption in Theorem 1.8.

Thus there exist $N \geq 1,0<c_{1}<\cdots<c_{N}, x_{1}^{+}, \ldots, x_{N}^{+} \in \mathbb{R}$, and a possibly smaller $\gamma>0$ such that

$$
\left\|u_{d}(t)-\sum_{i=1}^{N} R_{c_{i}, x_{i}^{+}}(t)\right\|_{H^{1}}=\mathrm{O}\left(e^{-\gamma t}\right), \quad \text { as } t \rightarrow+\infty .
$$

Claim 4.2. We have

$$
\left\|u(t)-\sum_{i=1}^{N} R_{c_{i}, x_{i}^{+}}(t)\right\|_{L^{2}}=\mathrm{O}\left(t^{-\frac{1}{3}}\right), \quad \text { as } t \rightarrow+\infty .
$$

Proof of Claim 4.2. Consider $\beta \in\left(0, \min \left\{c_{1}, \rho\right\}\right)$ so that (4.2) is guaranteed for some $\gamma>0$ and so that (by the non dispersion assumption and the sech-shaped profiles of the solitons $\left.R_{c_{i}, x_{i}^{+}}\right)$

$$
\|u(t)\|_{L^{2}(x \leq \beta t)}+\sum_{i=1}^{N}\left\|R_{c_{i}, x_{i}^{+}}(t)\right\|_{L^{2}(x \leq \beta t)}=\mathrm{O}\left(e^{-\gamma t}\right)
$$


even if it means reducing $\gamma>0$. We perform then

$$
\begin{aligned}
\left\|u(t)-u_{d}(t)\right\|_{L^{2}} & =\left\|u(t)-u_{d}(t)\right\|_{L^{2}(x \leq \beta t)}+\left\|u(t)-u_{d}(t)\right\|_{L^{2}(x>\beta t)} \\
& \leq\|u(t)\|_{L^{2}(x \leq \beta t)}+\left\|u_{d}(t)\right\|_{L^{2}(x \leq \beta t)}+\mathrm{O}\left(t^{-\frac{1}{3}}\right) \\
& \leq\left\|u_{d}(t)\right\|_{L^{2}(x \leq \beta t)}+\mathrm{O}\left(t^{-\frac{1}{3}}+e^{-\gamma t}\right) \\
& \leq\left\|u_{d}(t)-\sum_{i=1}^{N} R_{c_{i}, x_{i}^{+}}(t)\right\|_{H^{1}}+\sum_{i=1}^{N}\left\|R_{c_{i}, x_{i}^{+}}(t)\right\|_{L^{2}(x \leq \beta t)}+\mathrm{O}\left(t^{-\frac{1}{3}}\right) \\
& =\mathrm{O}\left(t^{-\frac{1}{3}}\right),
\end{aligned}
$$

by the embeddings $H^{1}(\mathbb{R}) \hookrightarrow H^{1}(x \leq \beta t) \hookrightarrow L^{2}(x \leq \beta t)$ and by (4.5). By means of the triangular inequality and once again (4.5), we deduce the expected estimate in Claim 4.2.

We are now able to finish the proof of Theorem 1.8. Indeed, let us make the following key observation.

Claim 4.3. The solution u belongs to $L^{\infty}\left([0,+\infty), H^{2}(\mathbb{R})\right)$.

Proof of Claim 4.3. This is an immediate consequence of the following conservation law for the KdV equation

$$
\frac{d}{d t} \int_{\mathbb{R}}\left\{\left(\partial_{x}^{2} u\right)^{2}-\frac{10}{3}\left(\partial_{x} u\right)^{2} u+\frac{5}{9} u^{4}\right\}(t, x) d x=0,
$$

of the Sobolev embedding $H^{1}(\mathbb{R}) \hookrightarrow L^{\infty}(\mathbb{R})$, and from the fact that $u$ belongs to $L^{\infty}\left(\mathbb{R}, H^{1}(\mathbb{R})\right)$.

As a consequence of Claim $4.3, v$ belongs also to $L^{\infty}\left(\left[T_{1},+\infty\right), H^{2}(\mathbb{R})\right)$. Then, integrating by parts and using the Cauchy-Schwarz inequality and Claim 4.2, we infer that

$$
\int_{\mathbb{R}}\left(\partial_{x} v\right)^{2}(t) d x=-\int_{\mathbb{R}} v(t) \partial_{x}^{2} v(t) d x \leq\|v(t)\|_{L^{2}}\|v(t)\|_{H^{2}} \leq C t^{-\frac{1}{3}},
$$

from which it results that

$$
\left\|u(t)-\sum_{i=1}^{N} R_{c_{i}, x_{i}^{+}}(t)\right\|_{H^{1}} \rightarrow 0, \quad \text { as } t \rightarrow+\infty .
$$

Hence $u$ is a multi-soliton in $+\infty$. By means of the well-known theory concerning multisolitons of the KdV equation (see for instance Miura [27]), we deduce that $u$ is also a multisoliton in $-\infty$. This ends the proof of Theorem 1.8.

4.2. Non dispersive solutions of the modified Korteweg-de Vries equation. Theorem 1.10 is obtained by using the same strategy as that developed in the previous subsection. Thus we will only sketch its proof.

As for the KdV case, we apply first the following decomposition result, obtained from [30, Chapter 5, Theorem 5.1] and from [3, Theorem 1.10] where a more precise version can be found.

Theorem 4.4 (Schuur [30], Chen and Liu [3]). Let $p=3$ and $u_{0} \in \mathscr{C}^{4}(\mathbb{R})$ be such that for some $C_{0}>0$, for all $k=0, \ldots, 4$, and for all $x \in \mathbb{R}$,

$$
\left|\frac{\partial^{k} u_{0}(x)}{\partial x^{k}}\right| \leq C_{0}|x|^{-11}
$$

and be generic as in Theorem 1.10, with scattering data (1.12). Let $u$ be the corresponding global solution of $(m K d V)$. 
Then there exist signs $\epsilon_{i}= \pm 1, i=1, \ldots, N_{1}$, and parameters $x_{0, i}, i=1, \ldots, N_{1}$, and $x_{1, j}$, $x_{2, j}, j=1, \ldots, N_{2}$, such that for all $v_{+}>0$ and $v_{-}<0$, there exists $K \geq 0$ such that for all $t>0$, denoting

$$
P(t):=\sum_{i=1}^{N_{1}} \epsilon_{i} R_{2 c_{i}, x_{0, i}}(t)+\sum_{j=1}^{N_{2}} B_{\sqrt{2} \alpha_{j}, \sqrt{2} \beta_{j}, x_{1, j}, x_{2, j}}(t),
$$

we have

$$
\|u(t)-P(t)\|_{L^{\infty}\left(x>v_{+} t\right)}+\|u(t)-P(t)\|_{L^{2}\left(x>v_{+} t\right)} \leq K t^{-\frac{1}{3}},
$$

and

$$
\|u(t)-P(t)\|_{L^{\infty}\left(x<v_{-} t\right)} \leq K t^{-\frac{1}{2}}
$$

Then, the non dispersion assumption (1.6) in Theorem 1.10 shows that $N_{1}+N_{2} \geq 1$. Since the profiles of the breathers are sech-shaped, due to (1.6) and (4.10), we deduce that the breathers have positive (envelope) velocities.

Now, proceeding as in the proof of Claim 4.2, we obtain in fact that

$$
\|u(t)-P(t)\|_{L^{2}}=\mathrm{O}\left(t^{-\frac{1}{3}}\right) .
$$

Moreover, $(\mathrm{mKdV})$ admits conservation laws of orders 2, 3, and 4 in the spirit of (4.6), which shows that $u$ belongs to $L^{\infty}\left([0,+\infty), H^{4}(\mathbb{R})\right)$. Proceeding similarly to subsection 4.1 , we obtain that

$$
\|u(t)-P(t)\|_{H^{2}} \rightarrow 0, \quad \text { as } t \rightarrow+\infty .
$$

Finally, by the uniqueness and smoothness results and the estimates in higher Sobolev spaces proven by Semenov [31] as far as multi-breathers are concerned, we deduce that $u$ belongs to $\mathscr{C}\left([0,+\infty), H^{s}(\mathbb{R})\right)$ and that there exist $\gamma>0$ and positive constants $C_{s}$ such that for all $s \in \mathbb{N}$,

$$
\|u(t)-P(t)\|_{H^{s}} \leq C_{s} e^{-\gamma t}, \quad \text { as } t \rightarrow+\infty .
$$

This finishes proving Theorem 1.10

\section{Appendix: Proof of Proposition 3.6}

The proof follows the same lines as that of Proposition 3 and paragraph 3.2 in Martel [12] for the $L^{2}$-subcritical and critical cases, and that of Lemma 4.1 in Combet [5] for the supercritical case. For the sake of simplicity and for the reader's convenience, we present here the essential ideas and also the changes in the $L^{2}$-subcritical case only.

Remark 5.1. We mention that in the $L^{2}$-critical and supercritical cases, the proof is basically changed in terms of the coercivity property we use to control the modulation function $\epsilon$ defined below in Step 1. The monotonicity properties of local mass and energy obtained in Step 2 are still valid in these cases.

Concerning the critical case, the idea is to modulate the scaling parameter in addition to the translation parameter so as to ensure a second orthogonality condition satisfied by $\epsilon$, namely $\int_{\mathbb{R}} \epsilon(t) \tilde{R}_{j}(t)^{3} d x=0$, and then to apply a localized version of the coercivity property available in this case, which leads to:

$$
\exists \lambda_{0}>0, \forall t, \quad\|\epsilon(t)\|_{H^{1}}^{2} \leq \lambda_{0} \mathcal{H}(t),
$$

with $\mathcal{H}$ defined in Step 3.

In the supercritical case, it is known from Pego and Weinstein [29] that, considering the standard linearized operator $L$ on $H^{1}(\mathbb{R})$ defined by $L v:=-\partial_{x}^{2} v+v-p Q^{p-1} v$, the composed operator $L \partial_{x}$ has two eigenfunctions $Z^{+}$and $Z^{-}$related by $Z^{-}(x)=Z^{+}(-x)$, which decay 
exponentially, and such that $L \partial_{x} Z^{ \pm}= \pm e_{0} Z^{ \pm}$for some $e_{0}>0$. In this case, we only have to make modifications in Step 3 by using this time

$$
\exists \lambda_{0}>0, \forall t, \quad\|\epsilon(t)\|_{H^{1}}^{2} \leq \lambda_{0} \mathcal{H}(t)+\frac{1}{\lambda_{0}} \sum_{i, \pm}\left(\int_{\mathbb{R}} \epsilon(t) \tilde{Z}_{i}^{ \pm}(t) d x\right)^{2},
$$

where $\tilde{Z}_{i}^{ \pm}(t):=Z_{i}^{ \pm}\left(\cdot-x_{i}(t)-y_{i}(t)\right)$ and $Z_{i}^{ \pm}(x):=c_{i}^{-\frac{1}{2}} Z^{ \pm}\left(c_{i}^{\frac{1}{2}} x\right)$.

(The functions $y_{i}$ are defined in Lemma 5.2 below.) The control of $\int_{\mathbb{R}} \epsilon(t) \tilde{Z}_{i}^{ \pm}(t) d x$ by a function of $t$ which decreases with exponential speed follows the strategy of Combet (for full details, see [5, paragraph 4.1 Step 4]).

Step 1: Set up of a modulation argument

Set $v:=\min \left\{c_{1}, \delta_{0}\right\}$. We claim the following

Lemma 5.2. There exist $T \geq 0$ and $\alpha_{1} \in(0,1]$ such that for all $\tilde{\alpha} \leq \alpha_{1}$, the following holds. There exist unique $\mathscr{C}^{1}$ functions $y_{i}:[T,+\infty) \rightarrow \mathbb{R}$ such that defining

$$
\epsilon:=u-\sum_{i=1}^{N} \tilde{R}_{i}
$$

where $\tilde{R}_{i}(t, x):=Q_{c_{i}}\left(x-x_{i}(t)-y_{i}(t)\right)$, we have for all $t \geq T$,

$$
\forall i \in\{1, \ldots, N\}, \quad \int_{\mathbb{R}} \epsilon\left(\tilde{R}_{i}\right)_{x}(t) d x=0 .
$$

In addition, there exists $K>0$ such that for all $t \geq T$, for all $i \in\{1, \ldots, N\}$,

$$
\begin{gathered}
\|\epsilon(t)\|_{H^{1}}+\sum_{i=1}^{N}\left|y_{i}(t)\right| \leq K \tilde{\alpha} \\
\left|x_{i}^{\prime}(t)+y_{i}^{\prime}(t)-c_{i}\right| \leq K\left(\int_{\mathbb{R}} \epsilon^{2}(t) e^{-\sqrt{v}\left|x-x_{i}(t)\right|} d x\right)^{\frac{1}{2}}+K e^{-\frac{1}{4} v^{\frac{3}{2}} t} .
\end{gathered}
$$

Proof. Recall that the proof of existence and uniqueness of the functions $y_{i}(t)$ is based on the implicit function theorem. We refer to [26, proof of Lemma 8] and also to [15, paragraph 2.3] for a complete proof in the case of one soliton. Moreover, estimate (5.6) which involves $v \leq c_{1}$ is obtained formally by writing the equation of $\epsilon$, that is

$$
\epsilon_{t}+\partial_{x}^{3} \epsilon=\sum_{i=1}^{N}\left(x_{i}^{\prime}+y_{i}^{\prime}-c_{i}\right)\left(\tilde{R}_{i}\right)_{x}-\left(\left(\epsilon+\sum_{i=1}^{N} \tilde{R}_{i}\right)^{p}-\sum_{i=1}^{N} \tilde{R}_{i}^{p}\right)_{x}
$$

by multiplying it by $\left(\tilde{R}_{i}\right)_{x}$, and by using the following properties:

$$
0=\frac{d}{d t} \int_{\mathbb{R}} \epsilon\left(\tilde{R}_{i}\right)_{x} d x=\int_{\mathbb{R}} \partial_{t} \epsilon \partial_{x} \tilde{R}_{i} d x-\left(x_{i}^{\prime}+y_{i}^{\prime}\right) \int_{\mathbb{R}} \epsilon \partial_{x}^{2} \tilde{R}_{i} d x
$$

$\forall i \neq j, \forall t \geq T_{2}$,

$$
\begin{gathered}
\left|\tilde{R}_{i}(t, x)\right|+\left|\partial_{x} \tilde{R}_{i}(t, x)\right| \leq C e^{-\sqrt{v}\left|x-x_{i}(t)\right|} \\
\int_{\mathbb{R}}\left\{\tilde{R}_{i}(t, x) \tilde{R}_{j}(t, x)+\left|\partial_{x} \tilde{R}_{i}(t, x) \partial_{x} \tilde{R}_{j}(t, x)\right|\right\} d x \leq C e^{-\frac{v^{\frac{3}{2}}}{2} t} .
\end{gathered}
$$

Note that (5.10) is a consequence of the decoupling assumption (3.16). We refer to [12] and the references therein for more details. 
Step 2: Monotonicity properties for localized mass and some modified energy of $u$

Let $\psi: x \mapsto \frac{2}{\pi} \operatorname{Arctan}\left(e^{-\frac{\sqrt{v}}{2} x}\right)$ be defined on $\mathbb{R}$ so that for all $x \in \mathbb{R}$,

$$
\psi^{\prime}(x) \leq 0, \quad\left|\psi^{\prime}(x)\right| \leq \frac{\sqrt{v}}{\pi} e^{-\frac{\sqrt{v}}{2}|x|}, \quad\left|\psi^{(3)}(x)\right| \leq \frac{v}{4}\left|\psi^{\prime}(x)\right|
$$

(we recall $v=\min \left\{c_{1}, \delta_{0}\right\}$ ). Then define on $\mathbb{R}^{+} \times \mathbb{R}$ :

$$
\forall i \in\{1, \ldots, N-1\}, \quad \psi_{i}:(t, x) \mapsto \psi\left(x-\frac{x_{i}(t)+x_{i+1}(t)}{2}\right), \quad \psi_{N}:(t, x) \mapsto 1
$$

and also

$$
\phi_{1}:=\psi_{1}, \quad \forall i \in\{2, \ldots, N-1\}, \quad \phi_{i}:=\psi_{i}-\psi_{i-1}, \quad \phi_{N}:=1-\psi_{N-1} .
$$

Remark 5.3. Note that by definition and by (3.16), for $t>0, \phi_{i}(t)$ takes values close to 1 in a neighborhood of $x_{i}(t)$ and takes values close to 0 around $x_{j}(t)$ for $j \neq i$.

Take $\kappa \in\left(0, \frac{c_{1}}{4}\right)$ and consider now for all $i \in\{1, \ldots, N-1\}$ the following quantities:

- (localized mass of $u$ at the left of $\frac{x_{i}(t)+x_{i+1}(t)}{2}$ )

$$
\mathcal{M}_{i}(t):=\int_{\mathbb{R}} u^{2}(t, x) \psi_{i}(t, x) d x
$$

- (modified localized energy of $u$ at the left of $\frac{x_{i}(t)+x_{i+1}(t)}{2}$ )

$$
\tilde{\mathcal{E}}_{i}(t):=\int_{\mathbb{R}}\left(\frac{1}{2} u_{x}^{2}-\frac{1}{p+1} u^{p+1}+\kappa u^{2}\right) \psi_{i}(t, x) d x .
$$

Let also

$$
\mathcal{M}_{N}(t):=\int_{\mathbb{R}} u^{2}(t, x) \psi_{N}(t, x) d x
$$

(which is nothing but the mass of $u$ ) and

$$
\tilde{\mathcal{E}}_{N}(t):=\int_{\mathbb{R}}\left(\frac{1}{2} u_{x}^{2}-\frac{1}{p+1} u^{p+1}+\kappa u^{2}\right) \psi_{N}(t, x) d x
$$

(which is a global quantity linked to the energy of $u$ ).

Remark 5.4. The reason why we have to choose $\kappa$ small enough appears clearly in Step 3 (see Remark 5.7).

We claim now a monotonicity result on the preceding quantities.

Lemma 5.5. There exist $T_{1} \geq T_{0}$ and $K_{1} \geq 0$ such that for all $t \geq T_{1}$ and for all $i \in\{1, \ldots, N\}$,

$$
\frac{d \mathcal{M}_{i}}{d t}(t) \geq-K_{1} e^{-\frac{v^{\frac{3}{2}}}{4} t} \quad \text { and } \quad \frac{d \tilde{\mathcal{E}}_{i}}{d t}(t) \geq-K_{1} e^{-\frac{v^{\frac{3}{2}}}{4} t} .
$$

Proof. First we observe that

$$
\frac{d \mathcal{M}_{i}}{d t}=-\int_{\mathbb{R}}\left(3 u_{x}^{2}+\frac{x_{i}^{\prime}+x_{i+1}^{\prime}}{2} u^{2}-\frac{2 p}{p+1} u^{p+1}\right) \psi_{i}^{\prime} d x+\int_{\mathbb{R}} u^{2} \psi_{i}^{(3)} d x .
$$

For all $\eta_{0}>0$, there exists $T_{\eta_{0}} \geq 0$ such that for all $t \geq T_{\eta_{0}}$,

$$
\left\|u(t)-\sum_{i=1}^{N} Q_{c_{i}}\left(\cdot-x_{i}(t)\right)\right\|_{H^{1}} \leq \eta_{0}
$$

and for all $R_{0}>0$, for each $(t, x) \in \mathbb{R}^{+} \times \mathbb{R}$ such that $x_{i}(t)+R_{0} \leq x \leq x_{i+1}(t)-R_{0}$, we have

$$
|u(t, x)| \leq \sum_{i=1}^{N} Q_{c_{i}}\left(x-x_{i}(t)\right)+C\left\|u(t)-\sum_{i=1}^{N} Q_{c_{i}}\left(\cdot-x_{i}(t)\right)\right\|_{H^{1}} \leq C \sum_{i=1}^{N} e^{-\sqrt{c_{i}} R_{0}}+\eta_{0} .
$$


NON DISPERSIVE SOLUTIONS OF THE GKDV EQUATIONS

21

Thus, for $R_{0}$ sufficiently large and for $\eta_{0}>0$ small enough being fixed, we have for some $T_{1}>T_{0}$ : for all $t \geq T_{1}$, for all $x \in\left[x_{i}(t)+R_{0}, x_{i+1}(t)-R_{0}\right]$,

$$
\frac{2 p}{p+1}|u(t, x)|^{p-1} \leq \frac{v}{4} \text {. }
$$

If $x>x_{i+1}(t)-R_{0}$ or $x<x_{i}(t)+R_{0}$, then

$$
\left|x-\frac{x_{i}(t)+x_{i+1}(t)}{2}\right|>\frac{x_{i+1}(t)-x_{i}(t)}{2}-R_{0}>\frac{v t}{2}-R_{0} .
$$

Consequently, for $t \geq T_{1}$ and $x \notin\left[x_{i}(t)+R_{0}, x_{i+1}(t)-R_{0}\right]$, we obtain

$$
\left|\psi_{i}^{\prime}(t, x)\right| \leq \frac{\sqrt{v}}{\pi} e^{-\frac{\sqrt{v}}{2}\left(\frac{x_{i+1}(t)-x_{i}(t)}{2}-R_{0}\right)} \leq C e^{-\frac{v^{\frac{3}{2}}}{4} t} .
$$

We deduce that

$$
\begin{aligned}
\frac{d \mathcal{M}_{i}}{d t}(t) & \geq-\int_{\mathbb{R}}\left(3 u_{x}^{2}+\left(\delta_{0}-\frac{v}{4}\right) u^{2}\right) \psi_{i}^{\prime}-\int_{\mathbb{R}} \frac{v}{4} u^{2}\left|\psi_{i}^{\prime}\right|-C e^{-\frac{v^{\frac{3}{2}}}{4} t} \\
& \geq-\int_{\mathbb{R}}\left(3 u_{x}^{2}+\frac{v}{2} u^{2}\right) \psi_{i}^{\prime}-C e^{-\frac{v^{\frac{3}{2}}}{4} t} \geq-C e^{-\frac{v^{\frac{3}{2}}}{4} t} .
\end{aligned}
$$

Similarly, we compute

$$
\begin{aligned}
\frac{d \tilde{\mathcal{E}}_{i}}{d t}= & -\int_{\mathbb{R}}\left[\left(u_{x x}^{2}+u^{p}\right)^{2}+2 u_{x x}^{2}+\frac{x_{i}^{\prime}+x_{i+1}^{\prime}}{2} u_{x}^{2}-\frac{x_{i}^{\prime}+x_{i+1}^{\prime}}{p+1} u^{p+1}\right]\left(\psi_{i}\right)_{x} d x \\
& +\int_{\mathbb{R}} u_{x}^{2} \psi_{i}^{(3)} d x+2 p \int_{\mathbb{R}} u_{x}^{2} u^{p-1}\left(\psi_{i}\right)_{x} d x+\kappa \frac{d \mathcal{M}_{i}}{d t} \\
\geq & -v \int_{\mathbb{R}} u_{x}^{2}\left(\psi_{i}\right)_{x}+\frac{v}{4} \int_{\mathbb{R}} u_{x}^{2}\left(\psi_{i}\right)_{x}+2 p \int_{\mathbb{R}} u_{x}^{2} u^{p-1}\left(\psi_{i}\right)_{x} \\
& -\kappa \int_{\mathbb{R}} 3 u_{x}^{2} \psi_{i}^{\prime}(x)-C e^{-\frac{v^{\frac{3}{2}}}{4} t}+\frac{x_{i}^{\prime}+x_{i+1}^{\prime}}{2} \int_{\mathbb{R}}\left(\frac{2}{p+1} u^{p+1}-\kappa u^{2}\right)\left(\psi_{i}\right)_{x} .
\end{aligned}
$$

As before, we can increase $T_{1}$ and reduce $\eta_{0}$ to have

$$
2 p\left|\int_{\mathbb{R}} u_{x}^{2} u^{p-1}\left(\psi_{i}\right)_{x}\right| \leq \frac{v}{4} \int_{\mathbb{R}} u_{x}^{2}\left|\left(\psi_{i}\right)_{x}\right|+C e^{-\frac{v^{\frac{3}{2}}}{4} t}
$$

and

$$
\left|\frac{2}{p+1} \int_{\mathbb{R}} u^{p+1}\left(\psi_{i}\right)_{x}\right| \leq \frac{\kappa}{2} \int_{\mathbb{R}} u^{2}\left|\left(\psi_{i}\right)_{x}\right| .
$$

Eventually, this leads to

$$
\begin{aligned}
\frac{d \tilde{\mathcal{E}}_{i}}{d t} & \geq-\frac{3}{4} v \int_{\mathbb{R}} u_{x}^{2}\left(\psi_{i}\right)_{x}-C e^{-\frac{v^{\frac{3}{2}}}{4} t}+\frac{\kappa\left(x_{i}^{\prime}+x_{i+1}^{\prime}\right)}{4} \int_{\mathbb{R}} u^{2}\left|\left(\psi_{i}\right)_{x}\right| \\
& \geq \frac{3}{4} v \int_{\mathbb{R}} u_{x}^{2}\left|\left(\psi_{i}\right)_{x}\right|+\frac{\kappa \delta_{0}}{2} \int_{\mathbb{R}} u^{2}\left|\left(\psi_{i}\right)_{x}\right|-C e^{-\frac{\sigma^{\frac{3}{2}}}{4} t} \geq-C e^{-\frac{v^{\frac{3}{2}}}{4} t} .
\end{aligned}
$$

Step 3: A Weinstein type functional

Let the functional $\mathcal{H}$ be given by

$$
\mathcal{H}:=\sum_{i=1}^{N} \frac{1}{c_{i}^{2}} \int_{\mathbb{R}}\left\{\partial_{x} \epsilon^{2}+c_{i} \epsilon^{2}-p \tilde{R}_{i}^{p-1} \epsilon^{2}\right\} \phi_{i},
$$

define

$$
\mathcal{F}:=\sum_{i=1}^{N} \frac{1}{c_{i}^{2}}\left\{\int_{\mathbb{R}}\left(\frac{1}{2} u_{x}^{2}-\frac{1}{p+1} u^{p+1}\right) \phi_{i}+\frac{c_{i}}{2} \int_{\mathbb{R}} u^{2} \phi_{i}\right\},
$$


and set $w(t):=u(t)-\sum_{i=1}^{N} R_{i}(t)$, where for all $i=1, \ldots, N$,

$$
R_{i}(t):=Q_{c_{i}}\left(\cdot-x_{i}(t)\right) .
$$

We gather next some properties satisfied by $\mathcal{H}$ and $\mathcal{F}$ which are essential to obtain $\|\epsilon(t)\|_{H^{1}}=$ $\mathrm{O}\left(e^{-\gamma t}\right)$ as $t \rightarrow+\infty$, for some $\gamma>0$.

Lemma 5.6. We have

(1) (coercivity property satisfied by $\mathcal{H}$ )

$$
\exists \lambda_{0}>0, \forall t \geq T, \quad\|\epsilon(t)\|_{H^{1}}^{2} \leq \lambda_{0} \mathcal{H}(t)+\frac{1}{\lambda_{0}} \sum_{i=1}^{N}\left(\int_{\mathbb{R}} \epsilon(t) \tilde{R}_{i}(t)\right)^{2} ;
$$

(2) (expansion of $\mathcal{H})$

$$
\mathcal{H}=2\left(\mathcal{F}-\sum_{i=1}^{N} \frac{1}{c_{i}^{2}}\left\{\int_{\mathbb{R}}\left(\frac{1}{2}\left(\partial_{x} Q_{c_{i}}\right)^{2}-\frac{1}{p+1} Q_{c_{i}}^{p+1}\right)+\frac{c_{i}}{2} \int_{\mathbb{R}} Q_{c_{i}}^{2} \phi_{i}\right\}\right)+g,
$$

where $|g(t)| \leq C e^{-\frac{1}{4} v^{\frac{3}{2}} t}+C \tilde{\alpha}\|\epsilon(t)\|_{L^{2}}^{2}$;

(3) (second expression for $\mathcal{F}$ )

$$
\begin{aligned}
\mathcal{F}= & \sum_{i=1}^{N-1}\left\{\left(\frac{1}{c_{i}^{2}}-\frac{1}{c_{i+1}^{2}}\right) \tilde{\mathcal{E}}_{i}+\left(\frac{1}{c_{i}}-\frac{1}{c_{i+1}}\right)\left(\frac{1}{2}-\kappa\left(\frac{1}{c_{i}}+\frac{1}{c_{i+1}}\right)\right) \mathcal{M}_{i}\right\} \\
& +\frac{1}{c_{N}^{2}} \tilde{\mathcal{E}}_{N}+\frac{1}{c_{N}}\left(\frac{1}{2}-\frac{\kappa}{c_{N}}\right) \mathcal{M}_{N}
\end{aligned}
$$

(4) (consequence of the monotonicity properties) For all $t^{\prime} \geq t \geq T_{1}$, for all $i=1, \ldots, N$,

$$
\begin{gathered}
\mathcal{M}_{i}(t)-\int_{\mathbb{R}} Q_{c_{i}}^{2} \leq 2 \int_{\mathbb{R}} w R_{i}\left(t^{\prime}\right)+\int_{\mathbb{R}} w^{2} \phi_{i}\left(t^{\prime}\right)+C e^{-\frac{v^{\frac{3}{2}}}{4} t} \\
\tilde{\mathcal{E}}_{i}(t)-\int_{\mathbb{R}}\left\{\frac{1}{2}\left(\partial_{x} Q_{c_{i}}\right)^{2}-\frac{Q_{c_{i}}^{p+1}}{p+1}\right\} \leq \\
C\|w(t)\|_{L^{\infty}} \int_{\mathbb{R}} w^{2} \phi_{i}\left(t^{\prime}\right)-c_{i} \int_{\mathbb{R}} w R_{i}\left(t^{\prime}\right) \\
+\frac{1}{2} \int_{\mathbb{R}}\left(w_{x}^{2}-p R_{i}^{p-1} w^{2}\right) \phi_{i}\left(t^{\prime}\right)+C e^{-\frac{v^{\frac{3}{2}}}{4} t} .
\end{gathered}
$$

Proof of Lemma 5.6. We only give some indications, and particularly the key ingredients. Property (5.24) is obtained by Abel transformation. Now, we focus on the other lines.

Estimate (5.22) is a consequence of a localized version around $\phi_{i}$ of the coercivity property of the linearized operator around $Q_{c_{i}}$ (for all $i=1, \ldots, N$ ), which holds under the orthogonality conditions (5.4) satisfied by $\epsilon$; we refer to [26, proof of Lemma 4].

To prove (5.23), one has obviously to replace $\epsilon$ by its definition (5.3).

To finish with, integrate the almost monotonicity properties as expressed in Lemma 5.5 between $t$ and $t^{\prime}$ and use the expression of $u\left(t^{\prime}\right)$ in terms of $w\left(t^{\prime}\right)$ in order to obtain (5.25) and (5.26).

Let us mention furthermore that (5.22), (5.23), (5.25), and (5.26) rely all on classical inequalities used in studying quantities which are localized near the solitons, and which write in the present context as follows:

$$
\begin{aligned}
\forall i \neq j, & \left(R_{i}(t, x)+\left|\partial_{x} R_{i}(t, x)\right|\right) \phi_{j}(t, x) \leq C e^{-\frac{v^{\frac{3}{2}}}{4} t} e^{-\frac{\sqrt{v}}{4}\left|x-x_{i}(t)\right|} \\
\forall i, j, & \left(R_{i}(t, x)+\left|\partial_{x} R_{i}(t, x)\right|\right)\left|\partial_{x} \phi_{j}(t, x)\right| \leq C e^{-\frac{v^{\frac{3}{2}}}{4} t} e^{-\frac{\sqrt{v}}{4}\left|x-x_{i}(t)\right|} \\
\forall i, & R_{i}(t, x)\left(1-\phi_{i}(t, x)\right) \leq C e^{-\frac{v^{\frac{3}{2}}}{4} t} e^{-\frac{\sqrt{v}}{4}\left|x-x_{i}(t)\right|} .
\end{aligned}
$$


Let us now explain how to conclude the proof. Note that (5.23), (5.24), (5.25), and (5.26) lead to: $\forall t^{\prime} \geq t \geq T$,

$$
\begin{aligned}
\mathcal{H}(t) \leq & C e^{-\frac{v^{\frac{3}{2}}}{4} t}+|g(t)| \\
& +2 \sum_{i=1}^{N-1}\left(\frac{1}{c_{i}^{2}}-\frac{1}{c_{i+1}^{2}}\right)\left(C\left\|w\left(t^{\prime}\right)\right\|_{L^{\infty}} \int_{\mathbb{R}} w^{2} \phi_{i}\left(t^{\prime}\right)-c_{i} \int_{\mathbb{R}} w\left(t^{\prime}\right) R_{i}\left(t^{\prime}\right) \phi_{i}\left(t^{\prime}\right)\right. \\
& \left.\quad+\frac{1}{2} \int_{\mathbb{R}}\left\{\left(\partial_{x} w\right)^{2}\left(t^{\prime}\right)-p R_{i}^{p-1} w^{2}\left(t^{\prime}\right)\right\} \phi_{i}\left(t^{\prime}\right)\right) \\
& +2 \sum_{i=1}^{N-1}\left(\frac{1}{c_{i}}-\frac{1}{c_{i+1}}\right)\left(\frac{1}{2}-\kappa\left(\frac{1}{c_{i}}+\frac{1}{c_{i+1}}\right)\right)\left(2 \int_{\mathbb{R}} w R_{i}\left(t^{\prime}\right)+\int_{\mathbb{R}} w^{2} \phi_{i}\left(t^{\prime}\right)\right) \\
& +\frac{2}{c_{N}^{2}}\left(C\left\|w\left(t^{\prime}\right)\right\|_{L^{\infty}} \int_{\mathbb{R}} w^{2} \phi_{N}\left(t^{\prime}\right)+\frac{1}{2} \int_{\mathbb{R}}\left\{\left(\partial_{x} w\right)^{2}-p R_{N}^{p-1} w^{2}\left(t^{\prime}\right)\right\} \phi_{N}\left(t^{\prime}\right)\right) \\
& +\frac{2}{c_{N}}\left(\frac{1}{2}-\frac{\kappa}{c_{N}}\right)\left(2 \int_{\mathbb{R}} w R_{N}\left(t^{\prime}\right)+\int_{\mathbb{R}} w^{2} \phi_{N}\left(t^{\prime}\right)\right)-\frac{2}{c_{N}} \int_{\mathbb{R}} w R_{N}\left(t^{\prime}\right),
\end{aligned}
$$

that is to: $\forall t^{\prime} \geq t \geq T$,

$$
\begin{aligned}
\mathcal{H}(t) \leq \sum_{i=1}^{N} \frac{1}{c_{i}^{2}}\left\{\left(\partial_{x} w\right)^{2}+c_{i} w^{2}\left(t^{\prime}\right)-p R_{i}^{p-1}\left(t^{\prime}\right) w^{2}\left(t^{\prime}\right)\right\} \phi_{i}\left(t^{\prime}\right) \\
+C\left\|w\left(t^{\prime}\right)\right\|_{L^{\infty}}\left\|w\left(t^{\prime}\right)\right\|_{L^{2}}^{2}+C \tilde{\alpha}\|\epsilon(t)\|_{L^{2}}^{2}+C e^{-\frac{v^{\frac{3}{2}}}{4} t},
\end{aligned}
$$

where $C$ does not depend on $\tilde{\alpha}$.

Remark 5.7. Note that the monotonicity property (5.25) can indeed be used in the preceding estimates since $\kappa$ verifies $\kappa\left(\frac{1}{c_{i}}+\frac{1}{c_{i+1}}\right)<\frac{1}{2}$ and $\frac{\kappa}{c_{N}}<\frac{1}{2}$.

Assumption (3.17) tells us exactly that $\left\|w\left(t^{\prime}\right)\right\|_{H^{1}} \underset{t^{\prime} \rightarrow+\infty}{\longrightarrow} 0$, thus we obtain

$$
\forall t \geq T, \quad \mathcal{H}(t) \leq C e^{-\frac{v^{\frac{3}{2}}}{4} t}+C \alpha\|\epsilon(t)\|_{L^{2}}^{2} .
$$

Remark 5.8. Notice that it is important to consider $w$ instead of $\epsilon$ in estimates (5.25) and (5.26) to obtain (5.31), thus to improve the a priori control of $\mathcal{H}$ by $\mathrm{O}\left(\|\epsilon\|_{H^{1}}^{2}\right)$.

Then, by (5.22) and by the following estimate

$$
\sum_{i=1}^{N}\left(\int_{\mathbb{R}} \epsilon(t) \tilde{R}_{i}(t)\right)^{2} \leq C e^{-\frac{v^{\frac{3}{2}}}{4} t}\|\epsilon(t)\|_{L^{2}}+C \tilde{\alpha}\|\epsilon(t)\|_{L^{2}}^{2}
$$

(see Martel [12, Step 3]) for a proof), there exists $C_{0}>0$ such that for all $t \geq T$,

$$
\|\epsilon(t)\|_{H^{1}}^{2} \leq C e^{-\frac{v^{\frac{3}{2}}}{4} t}+C \tilde{\alpha}\|\epsilon(t)\|_{H^{1}}^{2}+C \sum_{i=1}^{N}\left(\int_{\mathbb{R}} \epsilon(t) \tilde{R}_{i}(t)\right)^{2} \leq C_{0} e^{-\frac{v^{\frac{3}{2}}}{4} t}+C_{0} \tilde{\alpha}\|\epsilon(t)\|_{H^{1}}^{2} .
$$

Now, due to the independence of $C_{0}$ with respect to $\tilde{\alpha}$, even if it means taking a smaller $\tilde{\alpha}$ so that $C_{0} \tilde{\alpha}<1$, we infer

$$
\forall t \geq T, \quad\|\epsilon(t)\|_{H^{1}}^{2} \leq C e^{-\frac{v^{\frac{3}{2}}}{4} t} .
$$

By (5.6), this implies that

$$
\forall t \geq T, \quad\left|x_{i}^{\prime}(t)+y_{i}^{\prime}(t)-c_{i}\right| \leq C e^{-\frac{v^{\frac{3}{2}}}{8} t},
$$


which leads to the existence of $y_{i} \in \mathbb{R}$ such that

$$
x_{i}(t)+y_{i}(t)-c_{i} t \underset{t \rightarrow+\infty}{\longrightarrow} y_{i}
$$

and

$$
\left|x_{i}(t)+y_{i}(t)-c_{i} t-y_{i}\right| \leq C e^{-\frac{v^{\frac{3}{2}}}{8} t} .
$$

Hence, using (5.33), the triangular inequality, the following estimate

$$
\left\|Q_{c_{i}}\left(\cdot-x_{i}(t)-y_{i}(t)\right)-Q_{c_{i}}\left(\cdot-c_{i} t-y_{i}\right)\right\|_{H^{1}} \leq C\left|x_{i}(t)+y_{i}(t)-c_{i} t-y_{i}\right|
$$

(which is a consequence of Lemma 5.9 below), and (5.34), we have

$$
\left\|u(t)-\sum_{i=1}^{N} Q_{c_{i}}\left(\cdot-c_{i} t-y_{i}\right)\right\|_{H^{1}} \leq C e^{-\frac{v^{\frac{3}{2}}}{8} t} .
$$

Lemma 5.9. For all $i=1, \ldots, N$, for all $r \geq 0$, and for all $s \in \mathbb{N}^{*}$,

$$
\left\|\partial_{x}^{s} Q_{c_{i}}(\cdot-r)-\partial_{x}^{s} Q_{c_{i}}\right\|_{L^{2}}^{2} \leq\left(\left\|\partial_{x}^{s+1} Q_{c_{i}}\right\|_{L^{2}}^{2}+\left(r+2 z_{s}\right)\left\|\partial_{x}^{s+1} Q_{c_{i}}\right\|_{L^{\infty}}^{2}\right) r^{2},
$$

where $z_{s}:=\max \left(\left(\partial_{x}^{s+1} Q_{c_{i}}\right)^{-1}(\{0\}) \cap \mathbb{R}_{+}^{*}\right)$.

Proof of Lemmma 5.9. By the mean value theorem, we have:

$$
\left\|\partial_{x}^{s} Q_{c_{i}}(\cdot-r)-\partial_{x}^{s} Q_{c_{i}}\right\|_{L^{2}}^{2}=\int_{\mathbb{R}}\left(\partial_{x}^{s+1} Q_{c_{i}}\left(\xi_{x}\right)\right)^{2} r^{2} d x,
$$

where $x-r \leq \xi_{x} \leq x$ for all $x \in \mathbb{R}$.

Now, split the preceding integral into three regions: $x \leq-z_{s},-z_{s} \leq x \leq z_{s}+r$, and $x \geq z_{s}+r$. In the first and third regions, use the monotonicity of $\left(\partial_{x}^{s+1} Q_{c_{i}}\right)^{2}$. We have:

$$
\begin{cases}\left(\partial_{x}^{s+1} Q_{c_{i}}\left(\xi_{x}\right)\right)^{2} \leq\left(\partial_{x}^{s+1} Q_{c_{i}}(x)\right)^{2} & \text { if } x \leq-z_{s} \\ \left(\partial_{x}^{s+1} Q_{c_{i}}\left(\xi_{x}\right)\right)^{2} \leq\left(\partial_{x}^{s+1} Q_{c_{i}}(x-r)\right)^{2} & \text { if } x \geq z_{s}+r .\end{cases}
$$

If $-z_{s} \leq x \leq z_{s}+r$, we have

$$
\left(\partial_{x}^{s+1} Q_{c_{i}}\left(\xi_{x}\right)\right)^{2} \leq\left\|\partial_{x}^{s+1} Q_{c_{i}}\right\|_{L^{\infty}}^{2} .
$$

Thus, we obtain:

$$
\left\|\partial_{x}^{s} Q_{c_{i}}(\cdot-r)-\partial_{x}^{s} Q_{c_{i}}\right\|_{L^{2}}^{2} \leq\left(\left\|\partial_{x}^{s+1} Q_{c_{i}}\right\|_{L^{2}\left(|x| \geq z_{s}\right)}^{2}+\left(r+2 z_{s}\right)\left\|\partial_{x}^{s+1} Q_{c_{i}}\right\|_{L^{\infty}}^{2}\right) r^{2},
$$

which puts an end to the proof.

At the stage of (5.35), it suffices to see a posteriori that $\delta_{0} \leq c_{1}$ in order to obtain exactly (3.18). Let us justify it briefly.

For all $i \in\{1, \ldots, N\}$, set $f_{i}(t, x):=Q_{c_{i}}\left(x-x_{i}(t)\right)-Q_{c_{i}}\left(x-c_{i} t-y_{i}\right)$.

From (3.17) and (5.35), we deduce

$$
\left\|\sum_{i=1}^{N} f_{i}(t)\right\|_{H^{1}} \rightarrow 0, \quad \text { as } t \rightarrow+\infty .
$$

Define $t h_{p}(x):=\tanh \left(\frac{p-1}{2} x\right)$. A direct computation yields

$$
\forall x \in \mathbb{R}, \quad Q^{\prime}(x)=-t h_{p}(x) Q(x),
$$


from which we have for all $i \in\{1, \ldots, N\}$

$$
\begin{aligned}
\partial_{x} f_{i}(t, x)= & -\sqrt{c_{i}} t h_{p}\left(\sqrt{c_{i}}\left(x-c_{i} t-y_{i}\right)\right) f_{i}(t, x) \\
& +\sqrt{c_{i}}\left(t h_{p}\left(\sqrt{c_{i}}\left(x-x_{i}(t)\right)\right)-t h_{p}\left(\sqrt{c_{i}}\left(x-c_{i} t-y_{i}\right)\right)\right) Q_{c_{i}}\left(x-x_{i}(t)\right) .
\end{aligned}
$$

By (5.36), we have in particular

$$
\int_{\mathbb{R}}\left(\sum_{i=1}^{N} \partial_{x} f_{i}(t, x)\right)^{2} d x \rightarrow 0, \quad \text { as } t \rightarrow+\infty .
$$

Now, it follows from Lebesgue's dominated convergence theorem that

$$
\left\|\sum_{i=1}^{N} \sqrt{c_{i}} f_{i}(t)\right\|_{L^{2}} \rightarrow 0, \quad \text { as } t \rightarrow+\infty .
$$

Combining this result with (5.36) and due to the fact that the speeds $c_{i}$ are distinct two by two, we obtain successively for $k$ describing the integers from $N-1$ to 1 :

$$
\left\|\sum_{i=1}^{k}\left(\sqrt{c_{i}}-\sqrt{c_{k+1}}\right) f_{i}(t)\right\|_{L^{2}} \rightarrow 0, \quad \text { as } t \rightarrow+\infty .
$$

Hence $\left\|f_{1}(t)\right\|_{L^{2}} \underset{t \rightarrow+\infty}{\longrightarrow} 0$ and even $\left\|f_{1}(t)\right\|_{H^{1}} \underset{t \rightarrow+\infty}{\longrightarrow} 0$ judging by the expression of $\partial_{x} f_{1}$. This implies $x_{1}(t)-c_{1} t-y_{1} \underset{t \rightarrow+\infty}{\longrightarrow} 0$. Now it is clear that condition $x_{1}^{\prime}(t) \geq \delta_{0}$ forces to have $c_{1} \geq \delta_{0}$.

\section{REFERENCES}

[1] Miguel A. Alejo and Claudio Muñoz. Nonlinear Stability of MKDV Breathers. Comm. Partial Differ. Equ., 324:233-262, 2013.

[2] Jerry L. Bona, Panagiotis E. Souganidis, and Walter A. Strauss. Stability and Instability of Solitary Waves of Korteweg-de Vries Type. Proceedings of the Royal Society of London. Series A, Mathematical and Physical Sciences, 411(1841):395-412, 1987.

[3] Gong Chen and Jiaqi Liu. Soliton resolution for the modified KdV equation, 2019.

[4] Amy Cohen. Existence and regularity for solutions of the Korteweg-de Vries equation. Arch. Ration. Mech. Anal., 71:143-175, 1979.

[5] Vianney Combet. Multi-soliton solutions for the supercritical gKdV equations. Comm. Partial Differ. Equ., 36(3):380-419, 2011

[6] Raphaël Côte, Yvan Martel, and Frank Merle. Construction of multi-soliton solutions for the $L^{2}$-supercritical gKdV and NLS equations. Rev. Mat. Iberoam., 27(1):273-302, 2011.

[7] Wiktor Eckhaus and Peter C. Schuur. The emergence of solutions for the generalized Korteweg-de Vries equation from arbitrary initial conditions. Math. Meth. Appl. Sci., 5:97-116, 1983

[8] Manoussos Grillakis, Jalal Shatah, and Walter Strauss. Stability theory of solitary waves in the presence of symmetry, I. J. Funct. Analysis, 74(1):160-197, 1987.

[9] Carlos E. Kenig, Gustavo Ponce, and Luis Vega. Well-posedness and scattering results for the generalized Korteweg-de Vries equation via the contraction principle. Comm. Pure Appl. Math., 46:527-620, 1993.

[10] Yang Lan. Blow-up solutions for $L^{2}$-supercritical gKdV equations with exactly k blow-up points. Nonlinearity, $30,022016$.

[11] Céline Laurent and Yvan Martel. Smoothness and Exponential Decay of $L^{2}$-Compact Solutions of the Generalized KdV Equations. Comm. Partial Differ. Equ., 29:157-171, 2005.

[12] Yvan Martel. Asymptotic N-soliton-like solutions of the subcritical and critical generalized Korteweg-de Vries equations. Am. J. Math., 127:1103-1140, 2005.

[13] Yvan Martel and Frank Merle. A Liouville theorem for the critical generalized Korteweg-de Vries equation. $J$. Math. Pures Appl., 79:339-425, 2000.

[14] Yvan Martel and Frank Merle. Asymptotic stability of solitons for subcritical gKdV equations. Arch. Ration. Mech. Anal., 157:219-254, 2001.

[15] Yvan Martel and Frank Merle. Instability of solitons for the critical generalized Korteweg-de Vries equation Geometric And Funct. Analysis, 11(1):74-123, 2001.

[16] Yvan Martel and Frank Merle. Blow up in finite time and dynamics of blow up solutions for the $L^{2}$-critical generalized KdV equation. Journal of the American Mathematical Society, 15, 012002. 
[17] Yvan Martel and Frank Merle. Nonexistence of blow-up solution with minimal $L^{2}$-mass for the critical gKdV equation. Duke Math. J., 115(2):385-408, 112002.

[18] Yvan Martel and Frank Merle. Asymptotic stability of solitons of the subcritical gKdV equations revisited. Nonlinearity, 18:55-80, 2005.

[19] Yvan Martel and Frank Merle. Asymptotic stability of solitons of the gKdV equations with general nonlinearity. Math. Ann., 341:391-427, 2008.

[20] Yvan Martel and Frank Merle. Description of two soliton collision for the quartic gKdV equation. Ann. Math., 174(2):757-857, 2011.

[21] Yvan Martel and Frank Merle. Inelastic interaction of nearly equal solitons for the quartic gKdV equation. Invent. Math., 183(3):563-648, 2011.

[22] Yvan Martel and Frank Merle. On the nonexistence of pure multi-solitons for the quartic gKdV equation. Int. Math. Res. Not. IMRN, (3):688-739, 2015.

[23] Yvan Martel, Frank Merle, and Pierre Raphaël. Blow up for the critical generalized Korteweg-de Vries equation. I: Dynamics near the soliton. Acta Math., 212(1):59-140, 2014.

[24] Yvan Martel, Frank Merle, and Pierre Raphaël. Blow up for the critical generalized Korteweg-de Vries equation. II: Minimal mass dynamics. Journal of the European Mathematical Society, 17(8):1855 - 1925, 2015.

[25] Yvan Martel, Frank Merle, and Pierre Raphaël. Blow up for the critical generalized Korteweg-de Vries equation. III: Exotic regimes. Annali della Scuola Normale Superiore di Pisa, XIV:575-631, 2015.

[26] Yvan Martel, Frank Merle, and Tai-Peng Tsai. Stability and asymptotic stability in the energy space of the sum of $N$ solitons for subcritical gKdV equations. Comm. Math. Phys, 231:347-373, 2002.

[27] Robert M. Miura. The Korteweg-de Vries Equation: A Survey of Results. SIAM Review, 18(3):412-459, 1976.

[28] Claudio Muñoz. On the inelastic 2-soliton collision for $\mathrm{gKdV}$ equations with general nonlinearity. Int. Math. Research Notices, 9:1624-1719, 2010.

[29] Robert L. Pego and Michael I. Weinstein. Asymptotic stability of solitary waves. Comm. Math. Phys., 164:305349, 1994.

[30] Peter C. Schuur. Asymptotic Analysis of Solitons Problems. Berlin: Springer-Verlag, 1986.

[31] Alexander Semenov. On the existence and uniqueness of multi-breathers of (mKdV). preprint.

[32] Michael I. Weinstein. Lyapunov stability of ground states of nonlinear dispersive evolution equations. Comm. Pure Appl. Math., 39:51-67, 1986.

Institut de Recherche Mathématique Avancée UMR 7501, Université de Strasbourg, Strasbourg, France E-mail address: friederich@math.unistra.fr 